\title{
Tobacco use, exposure to secondhand smoke, and cessation counseling among medical students: cross-country data from the Global Health Professions Student Survey (GHPSS), 2005-2008
}

\author{
Charles W Warren ${ }^{1 *}$, Dhirendra N Sinha ${ }^{2}$, Juliette Lee ${ }^{1}$, Veronica Lea ${ }^{1}$, Nathan R Jones ${ }^{3}$
}

\begin{abstract}
Background: GHPSS is a school-based survey that collects self-administered data from students in regular classroom settings. GHPSS produces representative data at the national or city level in each country. This study aims to investigate the prevalence of tobacco use, exposure to secondhand smoke, and cessation counseling among medical students using the GHPSS data.

Methods: The Global Health Professions Student Survey (GHPSS) was conducted among $3^{\text {rd }}$ year medical students in 47 countries and the Gaza Strip/West Bank from 2005-2008 to determine the prevalence of tobacco use and amount of formal training in cessation counseling.

Results: In 26 of the 48 sites, over 20\% of the students currently smoked cigarettes, with males having higher rates than females in 37 sites. Over $70 \%$ of students reported having been exposed to secondhand smoke in public places in 29 of 48 sites. The majority of students recognized that they are role models in society (over $80 \%$ in 42 of 48 sites), believed they should receive training on counseling patients to quit using tobacco (over $80 \%$ in 41 of 48 sites), but few reported receiving formal training (less than $40 \%$ in 46 of 48 sites).

Conclusion: Tobacco control efforts must discourage tobacco use among health professionals, promote smoke free workplaces, and implement programs that train medical students in effective cessation-counseling techniques.
\end{abstract}

\section{Background}

"Tobacco use presents a rare confluence of circumstances: 1 ) a highly significant health threat; 2 ) a disinclination among clinicians to intervene consistently; and 3) the presence of effective interventions," a recent publication concluded [1]. The World Health Organization (WHO) estimates globally over 1 billion people currently smoke tobacco; with approximately 5 million deaths a year attributed to tobacco [2]. If current trends continue, WHO estimates tobacco attributable mortality will exceed 8 million per year by 2030. A disproportionate share of the global tobacco burden falls on

\footnotetext{
* Correspondence: wcw1@cdc.gov

'Office on Smoking and Health, US Centers for Disease Control and

Prevention, Atlanta, GA, USA

Full list of author information is available at the end of the article
}

developing countries, where $84 \%$ of 1.3 billion current smokers reside.

A number of studies have found that smoking cessation rates increase after even brief or simple counseling by physicians [3-6]. These studies have shown that physicians are willing to provide counseling and patients who smoke are receptive to counseling but in many cases the counseling is not repeated at subsequent visits nor is follow-up done. In addition, barriers have been identified that reduce the willingness of physicians to provide patient counseling, including time constraints during the consultation, physicians' lack of confidence in their ability to provide effective advice, and the smoking status of the physician.

In the WHO Framework Convention on Tobacco Control (WHO FCTC), the first public health treaty developed to counteract the global tobacco epidemic by 
encouraging countries to implement provisions to reduce the burden of tobacco, Article 12 calls on parties to implement effective and appropriate training on tobacco control to health workers including health professionals [7]. The 2008 WHO "MPOWER Report", a package of six tobacco policies proven to reduce the global tobacco epidemic, notes that "managing tobacco dependence is primarily the responsibility of a country's health-care system, including government, social security, NGOs and private clinical services" [2]. The Report identifies two primary interventions that will likely enhance quitting among tobacco users: 1) counseling by physicians and other health care workers during regular medical care (and quit line and community programs); and 2) access to low-cost pharmacological therapy [2].

Only a few studies have collected information on tobacco use, exposure to secondhand smoke (SHS), and training to provide cessation counseling among medical students. These studies used different sampling methods, questionnaires, and data collection procedures, and very few are from low or middle-income countries [8]. WHO, U.S. Centers for Disease Control and Prevention, and the Canadian Public Health Association have attempted to overcome these limitations by developing and implementing the Global Health Professions Student Survey (GHPSS) [9]. The data reported in this study come from GHPSS conducted among $3^{\text {rd }}$ year medical students in 47 countries and the Gaza Strip/ West Bank (identified as "sites" for the remainder of this paper).

\section{Methods}

The Medical GHPSS is part of the Global Tobacco Surveillance System, which collects data through four surveys: the Global Youth Tobacco Survey, the Global School Personnel Survey, the Global Adult Tobacco Survey, and GHPSS. GHPSS is a school-based survey of 3rd year students pursuing advanced degrees in dentistry, medicine, nursing or pharmacy. GHPSS has a standardized methodology for selecting schools, uniform data processing procedures and uses a core questionnaire [9].

The Medical GHPSS used two sample designs depending upon the total enrollment of $3^{\text {rd }}$ year students and the number of schools. In sites where the number of medical students exceeded 1000 and the number of schools was greater than 10, a representative sample of schools was selected probability proportionate to size, and all $3^{\text {rd }}$ year medical students in the schools were included. Sites with a sample of medical students included: Egypt, Bolivia, Mexico, Peru, Bangladesh, India, Indonesia, Nepal, and South Korea. For all other sites a census of schools and students was conducted where all schools and all 3rd year medical students were included in the survey (Table 1).
All sites were nominated by their Ministries' of Health in conjunction with their respective WHO regional offices. The majority of the 48 sites included in this study were low to middle income countries. The Medical GHPSS was conducted in schools during regular lectures and class sessions. Anonymous, self-administered data collection procedures were used. For all sites that conducted a census, a finite population correction factor was applied to take into account non-response and used in the variance of the estimates. For sites that did not conduct a census, a weighting factor was applied to each student record to adjust for nonresponse and variation in the probability of selection. SUDAAN was used to calculate weighted prevalence estimates and standard errors (SE) of the estimates (95\% confidence intervals (CI) were calculated from the SEs) [10]. T-tests were used to determine differences between subpopulations $[11,12]$. In this paper, differences in proportions are considered statistically significant if the t-test $p$ value was less than 0.05 . Results in this report are presented by WHO region with select countries highlighted. The six WHO regions are the African Region (AFR), the Eastern Mediterranean Region (EMR), the European Region (EUR), the Americas Region (AMR), the South East Asian Region (SEAR), and the Western Pacific Region (WPR). Data were not aggregated at the WHO regional level as the 47 countries represent only a fraction of the total Member States in each region: AFR 4 of 46; AMR 13 of 35; EMR 9 of 12; EUR 11 of 53; SEAR 7 of 11; and WPR 3 of 27. Data presented here are an expansion on previous published data [9].

For sites conducting the Medical GHPSS, the school response rate was $100 \%$ in 38 of the 48 sites; the classroom response rate was $100 \%$ in all sites; the student response rate ranged from $50.8 \%$ to $100 \%$; and the overall response rate ranged from $43.9 \%$ to $100 \%$ (Table 1 ). The number of students who participated in each survey varied due to the number of schools and students in each sample design.

This report includes information on current cigarette smoking, defined as those who answered one or more days to the question, "During the past 30 days (one month), on how many days did you smoke cigarettes?", current use of tobacco products other than cigarettes, defined as those who answered one or more days to the question, "During the past 30 days (one month), on how many days did you use chewing tobacco, snuff, bidis, cigars, or pipes (adapted to fit each country)?", exposure to SHS at home and in public places, official school policies banning smoking in school buildings and clinics, and policy enforcement. Additionally, attitude questions were asked regarding: health professionals as role models for their patients, whether health professionals think they should get training in patient cessation techniques, 
Table 1 Response Rates by Region and Country, Medical Global Health Professions Student Survey, 2005-2008

\begin{tabular}{|c|c|c|c|c|c|c|}
\hline $\begin{array}{l}\text { Country } \\
\text { (Site) }\end{array}$ & Year & $\begin{array}{c}\text { School Response } \\
\text { Rate (\%) }\end{array}$ & $\begin{array}{l}\text { Class Response } \\
\text { Rate (\%) }\end{array}$ & $\begin{array}{c}\text { Student Response } \\
\text { Rate (\%) }\end{array}$ & $\begin{array}{l}\text { Overall Response } \\
\text { Rate (\%) }\end{array}$ & $\begin{array}{c}\text { Number of 3rd Year } \\
\text { Students }\end{array}$ \\
\hline \multicolumn{7}{|c|}{ AFRICAN REGION (AFR) } \\
\hline Algeria & 2007 & 100.0 & 100.0 & 78.0 & 78.0 & 1,088 \\
\hline Kenya & 2008 & 100.0 & 100.0 & 73.0 & 73.0 & 227 \\
\hline Niger & 2008 & 80.0 & 100.0 & 77.7 & 62.1 & 754 \\
\hline Uganda & 2005 & 100.0 & 100.0 & 85.3 & 85.3 & 154 \\
\hline \multicolumn{7}{|c|}{ EASTERN MEDITERRANEAN REGION (EMR) } \\
\hline Egypt* & 2005 & 100.0 & 100.0 & 93.9 & 93.9 & 1,770 \\
\hline $\begin{array}{l}\text { Gaza Strip/West } \\
\text { Bank }\end{array}$ & 2007 & 100.0 & 100.0 & 92.3 & 92.3 & 81 \\
\hline $\operatorname{Iran}$ & 2007 & 90.2 & 100.0 & 50.8 & 45.9 & 1,065 \\
\hline Iraq & 2005 & 100.0 & 100.0 & 81.3 & 81.3 & 247 \\
\hline Lebanon & 2006 & 100.0 & 100.0 & 70.0 & 70.0 & 254 \\
\hline $\begin{array}{l}\text { Libyan Arab } \\
\text { Jamahiriya }\end{array}$ & 2006 & 90.0 & 100.0 & 77.9 & 70.1 & 1,488 \\
\hline Saudi Arabia & 2006 & 100.0 & 100.0 & 62.6 & 62.6 & 481 \\
\hline Sudan & 2007 & 100.0 & 100.0 & 64.5 & 64.5 & 591 \\
\hline $\begin{array}{l}\text { Syrian Arab } \\
\text { Republic }\end{array}$ & 2006 & 100.0 & 100.0 & 90.7 & 90.7 & 1,170 \\
\hline Tunisia & 2007 & 100.0 & 100.0 & 68.2 & 68.2 & 686 \\
\hline \multicolumn{7}{|c|}{ EUROPEAN REGION (EUR) } \\
\hline Albania & 2005 & 100.0 & 100.0 & 93.2 & 93.2 & 136 \\
\hline Armenia & 2006 & 100.0 & 100.0 & 90.0 & 90.0 & 177 \\
\hline $\begin{array}{l}\text { Bosnia \& } \\
\text { Herzegovina }\end{array}$ & 2006 & 100.0 & 100.0 & 94.4 & 94.4 & 421 \\
\hline Croatia & 2005 & 100.0 & 100.0 & 98.5 & 98.5 & 395 \\
\hline Czech Republic & 2006 & 100.0 & 100.0 & 94.7 & 94.7 & 690 \\
\hline Kyrgyzstan & 2008 & 100.0 & 100.0 & 92.7 & 92.7 & 112 \\
\hline Lithuania & 2006 & 100.0 & 100.0 & 64.6 & 64.6 & 221 \\
\hline $\begin{array}{l}\text { Russian } \\
\text { Federation }\end{array}$ & 2006 & 77.8 & 100.0 & 100.0 & 77.8 & 1,797 \\
\hline Serbia & 2006 & 100.0 & 100.0 & 81.6 & 81.6 & 775 \\
\hline Slovakia & 2006 & 100.0 & 100.0 & 91.9 & 91.9 & 378 \\
\hline Slovenia & 2007 & 100.0 & 100.0 & 69.9 & 69.9 & 142 \\
\hline \multicolumn{7}{|c|}{ REGION OF THE AMERICAS (AMR) } \\
\hline Argentina & 2005 & 100.0 & 100.0 & 85.9 & 85.9 & 306 \\
\hline Bolivia* & 2006 & 100.0 & 100.0 & 97.1 & 97.1 & 2,380 \\
\hline $\begin{array}{l}\text { Brazil (Rio de } \\
\text { Janeiro) }\end{array}$ & 2006 & 100.0 & 100.0 & 78.5 & 78.5 & 761 \\
\hline Chile & 2008 & 95.7 & 100.0 & 82.8 & 79.2 & 1,075 \\
\hline Costa Rica & 2006 & 100.0 & 100.0 & 100.0 & 100.0 & 279 \\
\hline Cuba (Havana) & 2008 & 100.0 & 100.0 & 80.2 & 80.2 & 477 \\
\hline Guatemala & 2008 & 100.0 & 100.0 & 72.3 & 72.3 & 351 \\
\hline Jamaica & 2008 & 100.0 & 100.0 & 65.5 & 65.5 & 107 \\
\hline$\overline{\text { Mexico* }}$ & 2006 & 93.3 & 100.0 & 83.4 & 77.8 & 2,226 \\
\hline Panama & 2008 & 100.0 & 100.0 & 85.7 & 85.7 & 285 \\
\hline Paraguay & 2008 & 100.0 & 100.0 & 89.1 & 89.1 & 396 \\
\hline$\overline{\text { Peru* }}$ & 2006 & 94.4 & 100.0 & 97.4 & 92.0 & 1,373 \\
\hline Uruguay & 2008 & 100.0 & 100.0 & 98.1 & 98.1 & 346 \\
\hline
\end{tabular}


Table 1 Response Rates by Region and Country, Medical Global Health Professions Student Survey, 2005-2008 (Continued)

\begin{tabular}{|c|c|c|c|c|c|c|}
\hline \multicolumn{7}{|c|}{ SOUTH-EAST ASIA REGION (SEAR) } \\
\hline Bangladesh* & 2006 & 100.0 & 100.0 & 77.0 & 77.0 & 777 \\
\hline India* & 2005 & 86.7 & 100.0 & 89.1 & 77.2 & 1,176 \\
\hline Indonesia* & 2006 & 100.0 & 100.0 & 77.4 & 77.4 & 1,580 \\
\hline Myanmar & 2006 & 100.0 & 100.0 & 87.5 & 87.5 & 1,430 \\
\hline Nepal* & 2005 & 83.3 & 100.0 & 74.9 & 62.5 & 327 \\
\hline Sri Lanka & 2006 & 100.0 & 100.0 & 78.0 & 78.0 & 617 \\
\hline Thailand & 2006 & 100.0 & 100.0 & 63.8 & 63.8 & 781 \\
\hline \multicolumn{7}{|c|}{ WESTERN PACIFIC REGION (WPR) } \\
\hline Cambodia & 2005 & 100.0 & 100.0 & 65.3 & 65.3 & 111 \\
\hline South Korea* & 2006 & 82.4 & 100.0 & 53.3 & 43.9 & 741 \\
\hline Viet Nam & 2007 & 100.0 & 100.0 & 88.9 & 88.9 & 1,423 \\
\hline
\end{tabular}

${ }^{*}$ sample of medical schools probability proportional to size.

and if they have ever received formal training on such cessation counseling techniques. The final country questionnaires were translated into local languages as needed and back-translated to check for accuracy. Cognitive lab testing of the questions was performed by an out-sourced independent survey company (RTI International).

\section{Ethical Considerations}

This study was a collaboration between the WHO region offices and $\mathrm{CDC}$ and the protocol was approved by both organizations. At CDC and WHO region offices, GHPSS was approved following ethical review procedures for surveillance systems. Detailed information about the study was provided verbally to the potential participants and informed consent was obtained from participating students. Data collection procedures assured confidentiality by the use of self-administered, anonymous questionnaires. Ethical approval from each of the participating universities was not required as the study was voluntary and confidentially was fully guaranteed.

\section{Results}

\section{Student Characteristics}

The percentage of medical students who were females was over $60 \%$ in 23 of the 48 sites (including all 11 sites in EUR); the percent female was less than $40 \%$ in 6 sites (Table 2). Over $90 \%$ of the students were less than age 25 in every site except Niger, Uganda, Lebanon, Bosnia \& Herzegovina, Argentina, Bolivia, Brazil, Costa Rica, Guatemala, Jamaica, Paraguay, and South Korea.

\section{Tobacco Use}

Among medical students, 3 sites had current smoking rates above 40\% (Albania, Bosnia \& Herzegovina, and
Bolivia) and 3 sites had rates less than 5\% (Uganda, Sri Lanka, and Thailand) (Table 3). Males were more likely than females to smoke cigarettes in 37 of 48 sites; females had higher rates than males in Serbia, Chile, and Thailand; and there were no gender differences in 8 of the 48 sites. For AFR, 37.7\% currently smoked cigarettes in Niger; while less than $10 \%$ smoked in the other 3 AFR sites. Current cigarette smoking ranged from over 20\% in Gaza Strip/West Bank (22.7\%) and Lebanon (28.2\%) to less than 10\% (Egypt, Iran, Sudan, and Tunisia) in EMR. In EUR, current cigarette smoking was over 30\% in every site, except Armenia, Czech Republic, Lithuania, and Slovenia. In AMR, current cigarette smoking was over $20 \%$ in all sites, except Brazil, Jamaica, and Panama. In the SEAR sites, current cigarette smoking was over $20 \%$ in Bangladesh and Nepal and less than 5\% in Sri Lanka and Thailand. In WPR, current cigarette smoking ranged from $16.0 \%$ in South Korea to $6.4 \%$ in Cambodia.

Among medical students, in the 4 AFR sites, current use of other tobacco products ranged from $27.7 \%$ in Niger to less than $5 \%$ in the other 3 AFR sites (Table 3). In EMR other tobacco use was over $20 \%$ in Lebanon and Syria but less than $10 \%$ in 6 of the 10 sites. In EUR, other tobacco use ranged from $18.0 \%$ in Serbia to less than $10 \%$ in 7 of the 11 sites. In AMR, use of other tobacco products was less than $10 \%$ in all sites, except Costa Rica (13.7\%), Guatemala (13.5\%), and Bolivia (10.6\%). Use of other tobacco products ranged from over $10 \%$ in Bangladesh, Myanmar, and Nepal to less than $10 \%$ in the other 4 SEAR sites. In WPR, use of other tobacco products was less than $5 \%$ in all 3 sites. Males were more likely than females to use other tobacco products in 38 of 48 sites; females had a higher rate than males in Iraq; and there was no gender difference in 9 sites. 
Table 2 Prevalence of Current Cigarette Smoking by Region and Country, Medical Global Health Professions Student Survey, 2005-2008 \& MPOWER 2008 Adult Data

\begin{tabular}{|c|c|c|c|c|}
\hline \multirow[b]{3}{*}{$\begin{array}{l}\text { Country } \\
\text { (Site) }\end{array}$} & \multicolumn{4}{|c|}{ Current Cigarette Smoking } \\
\hline & \multicolumn{2}{|c|}{ GHPSS Medical Students } & \multicolumn{2}{|c|}{ MPOWER 2008 Adult } \\
\hline & $\begin{array}{l}\text { Male \% } \\
(95 \% \mathrm{Cl})\end{array}$ & $\begin{array}{c}\text { Female \% } \\
(95 \% \mathrm{Cl})\end{array}$ & $\begin{array}{c}\text { Male \% } \\
(95 \% \mathrm{Cl})\end{array}$ & $\begin{array}{c}\text { Female \% } \\
(95 \% \mathrm{Cl})\end{array}$ \\
\hline \multicolumn{5}{|c|}{ AFRICAN REGION (AFR) } \\
\hline Algeria & $\begin{array}{c}23.7 \\
(21.3-26.2) \\
\end{array}$ & $\begin{array}{c}3.0 \\
(2.5-3.7) \\
\end{array}$ & $\begin{array}{c}28.7 \\
(26.3-31.2) \\
\end{array}$ & $\begin{array}{c}0.2 \\
(0-0.4) \\
\end{array}$ \\
\hline Kenya & $\begin{array}{c}10.9 \\
(8.0-14.8)\end{array}$ & $\begin{array}{c}8.7 \\
(5.9-12.6)\end{array}$ & $\begin{array}{c}22.2 \\
(19.4-24.9)\end{array}$ & $\begin{array}{c}0.9 \\
(0.5-1.3)\end{array}$ \\
\hline Niger & $\begin{array}{c}42.2 \\
(37.9-46.7)\end{array}$ & $\begin{array}{c}15.0 \\
(9.6-22.8)\end{array}$ & NA & NA \\
\hline Uganda & $\begin{array}{c}4.1 \\
(2.7-6.3)\end{array}$ & 0 & $\begin{array}{c}15.7 \\
(13.6-17.8)\end{array}$ & $\begin{array}{c}1.2 \\
(0.8-1.5)\end{array}$ \\
\hline \multicolumn{5}{|c|}{ EASTERN MEDITERRANEAN REGION (EMR) } \\
\hline Egypt & $\begin{array}{c}12.9 \\
(9.9-16.5)\end{array}$ & $\begin{array}{c}1.2 \\
(0.5-3.0)\end{array}$ & $\begin{array}{c}22.7 \\
(20.7-24.7)\end{array}$ & $\begin{array}{c}3.5 \\
(2.5-4.5) \\
\end{array}$ \\
\hline Gaza Strip/West Bank & $\begin{array}{c}39.1 \\
(33.8-44.6) \\
\end{array}$ & $\begin{array}{c}9.4 \\
(6.8-12.8) \\
\end{array}$ & NA & NA \\
\hline $\operatorname{Iran}$ & $\begin{array}{c}11.6 \\
(9.0-14.9) \\
\end{array}$ & $\begin{array}{c}2.8 \\
(1.9-4.0) \\
\end{array}$ & $\begin{array}{c}21.4 \\
(17.5-25.4) \\
\end{array}$ & $\begin{array}{c}1.7 \\
(1.1-2.2) \\
\end{array}$ \\
\hline Iraq & $\begin{array}{c}20.4 \\
(17.2-24.1)\end{array}$ & $\begin{array}{c}15.1 \\
(12.5-18.2)\end{array}$ & $\begin{array}{c}25.2 \\
(21.1-29.3) \\
\end{array}$ & $\begin{array}{c}1.3 \\
(0.4-2.1) \\
\end{array}$ \\
\hline Lebanon & $\begin{array}{c}36.1 \\
(31.8-40.7)\end{array}$ & $\begin{array}{c}18 \\
(14.4-22.3)\end{array}$ & $\begin{array}{c}29 \\
(24.2-33.9)\end{array}$ & $\begin{array}{c}6.9 \\
(2.7-11.2)\end{array}$ \\
\hline Libyan Arab Jamahiriya & $\begin{array}{c}22.3 \\
(20.1-24.6)\end{array}$ & $\begin{array}{c}1.9 \\
(1.4-2.7)\end{array}$ & NA & NA \\
\hline Saudi Arabia & $\begin{array}{c}13.1 \\
(10.0-16.9)\end{array}$ & $\begin{array}{c}9.6 \\
(6.2-14.8)\end{array}$ & $\begin{array}{c}25.2 \\
(21.4-28.9)\end{array}$ & $\begin{array}{c}3.0 \\
(1.2-4.8)\end{array}$ \\
\hline Sudan & $\begin{array}{c}19.6 \\
(15.9-23.9) \\
\end{array}$ & $\begin{array}{c}1.3 \\
(0.8-2.3) \\
\end{array}$ & NA & NA \\
\hline Syrian Arab Republic & $\begin{array}{c}24.3 \\
(23.3-25.3)\end{array}$ & $\begin{array}{c}4.8 \\
(4.2-5.5)\end{array}$ & $\begin{array}{c}41.2 \\
(16.5-65.9)\end{array}$ & NA \\
\hline Tunisia & $\begin{array}{c}26.5 \\
(22.7-30.7)\end{array}$ & $\begin{array}{c}4.1 \\
(3.2-5.4)\end{array}$ & $\begin{array}{c}47.4 \\
(44.7-50.1)\end{array}$ & $\begin{array}{c}1.0 \\
(0.6-1.4)\end{array}$ \\
\hline \multicolumn{5}{|c|}{ EUROPEAN REGION (EUR) } \\
\hline Albania & $\begin{array}{c}65.1 \\
(59.8-69.9)\end{array}$ & $\begin{array}{c}35.7 \\
(32.8-38.7)\end{array}$ & $\begin{array}{c}39.6 \\
(26.6-52.7)\end{array}$ & $\begin{array}{c}3.9 \\
(0.6-7.2)\end{array}$ \\
\hline Armenia & $\begin{array}{c}52.5 \\
(41.7-63.0)\end{array}$ & $\begin{array}{c}8.3 \\
(5.2-13.1)\end{array}$ & $\begin{array}{c}52.9 \\
(45.2-60.5)\end{array}$ & $\begin{array}{c}4.0 \\
(1.5-6.5)\end{array}$ \\
\hline Bosnia \& Herzegovina & $\begin{array}{c}45.0 \\
(43.0-47.0)\end{array}$ & $\begin{array}{c}37.8 \\
(36.4-39.2)\end{array}$ & $\begin{array}{c}48.8 \\
(42.3-55.3)\end{array}$ & $\begin{array}{c}32.0 \\
(26.3-37.8)\end{array}$ \\
\hline Croatia & $\begin{array}{c}35.9 \\
(31.5-40.4)\end{array}$ & $\begin{array}{c}37.1 \\
(34.1-40.3)\end{array}$ & $\begin{array}{c}37.5 \\
(35.7-39.3)\end{array}$ & $\begin{array}{c}25.4 \\
(24.3-26.5)\end{array}$ \\
\hline Czech Republic & $\begin{array}{c}26.2 \\
(21.9-30.9)\end{array}$ & $\begin{array}{c}19.8 \\
(17.2-22.5)\end{array}$ & $\begin{array}{c}35.9 \\
(29.4-42.5)\end{array}$ & $\begin{array}{c}23.4 \\
(16.4-30.3)\end{array}$ \\
\hline Kyrgyzstan & $\begin{array}{c}50.0 \\
(45.5-54.5)\end{array}$ & $\begin{array}{c}27.9 \\
(24.8-31.3)\end{array}$ & $\begin{array}{c}45 \\
(36.9-53.2)\end{array}$ & $\begin{array}{c}2.2 \\
(1.3-3.0)\end{array}$ \\
\hline Lithuania & $\begin{array}{c}48.6 \\
(39.7-57.7)\end{array}$ & $\begin{array}{c}19.5 \\
(15.8-23.8)\end{array}$ & $\begin{array}{c}44.4 \\
(37.3-51.5)\end{array}$ & $\begin{array}{c}17.6 \\
(15.0-20.2)\end{array}$ \\
\hline Russian Federation & $\begin{array}{c}46.1 \\
(44.2-48.1)\end{array}$ & $\begin{array}{c}34.9 \\
(33.5-36.2)\end{array}$ & $\begin{array}{c}70.2 \\
(59.2-81.3)\end{array}$ & $\begin{array}{c}23.2 \\
(16.7-29.7)\end{array}$ \\
\hline Serbia & $\begin{array}{c}31.2 \\
(28.7-33.8)\end{array}$ & $\begin{array}{c}36.7 \\
(34.9-38.6)\end{array}$ & $\begin{array}{c}41.4 \\
(36.5-46.3)\end{array}$ & $\begin{array}{c}40.4 \\
(35.7-45.1)\end{array}$ \\
\hline Slovakia & $\begin{array}{c}36.5 \\
(33.6-39.4)\end{array}$ & $\begin{array}{c}27.9 \\
(26.2-29.6)\end{array}$ & $\begin{array}{c}41.4 \\
(34.4-48.4)\end{array}$ & $\begin{array}{c}18.5 \\
(13.2-23.8)\end{array}$ \\
\hline
\end{tabular}


Table 2 Prevalence of Current Cigarette Smoking by Region and Country, Medical Global Health Professions Student Survey, 2005-2008 \& MPOWER 2008 Adult Data (Continued)

\begin{tabular}{|c|c|c|c|c|}
\hline Slovenia & $\begin{array}{c}22.5 \\
(15.9-30.8) \\
\end{array}$ & $\begin{array}{c}20.2 \\
(16.0-25.1) \\
\end{array}$ & $\begin{array}{c}29.6 \\
(23.6-35.5) \\
\end{array}$ & $\begin{array}{c}19.9 \\
(15.1-24.7) \\
\end{array}$ \\
\hline \multicolumn{5}{|c|}{ REGION OF THE AMERICAS (AMR) } \\
\hline Argentina & $\begin{array}{c}33.4 \\
(30.4-36.4)\end{array}$ & $\begin{array}{c}36.5 \\
(34.1-39.1)\end{array}$ & $\begin{array}{c}34.3 \\
(30.8-37.8)\end{array}$ & $\begin{array}{c}22.7 \\
(20.0-25.4)\end{array}$ \\
\hline Bolivia & $\begin{array}{c}50.3 \\
(43.9-56.7) \\
\end{array}$ & $\begin{array}{c}31.7 \\
(24.9-39.4) \\
\end{array}$ & $\begin{array}{c}35.7 \\
(27.8-43.6)\end{array}$ & $\begin{array}{c}27.3 \\
(24.0-30.6)\end{array}$ \\
\hline Brazil (Rio de Janeiro) & $\begin{array}{c}19.5 \\
(17.5-21.6)\end{array}$ & $\begin{array}{c}14.6 \\
(13.1-16.3)\end{array}$ & NA & NA \\
\hline Chile & $\begin{array}{c}27.1 \\
(25.5-28.9)\end{array}$ & $\begin{array}{c}29.3 \\
(27.5-31.3)\end{array}$ & $\begin{array}{c}42.2 \\
(33.7-50.7)\end{array}$ & $\begin{array}{c}30.1 \\
(25.2-35.0)\end{array}$ \\
\hline Costa Rica & 38.6 & 29.3 & $\begin{array}{c}26.7 \\
(22.5-30.9)\end{array}$ & $\begin{array}{c}7.3 \\
(5.8-8.9)\end{array}$ \\
\hline Cuba (Havana) & $\begin{array}{c}41.1 \\
(38.0-44.3) \\
\end{array}$ & $\begin{array}{c}21.2 \\
(19.1-23.5) \\
\end{array}$ & $\begin{array}{c}37.0 \\
(22.4-51.6) \\
\end{array}$ & $\begin{array}{c}27.3 \\
(20.8-33.8)\end{array}$ \\
\hline Guatemala & $\begin{array}{c}27.5 \\
(23.4-32.1) \\
\end{array}$ & $\begin{array}{c}17.8 \\
(14.6-21.6) \\
\end{array}$ & $\begin{array}{c}24.8 \\
(20.8-28.9) \\
\end{array}$ & $\begin{array}{c}3.9 \\
(3.0-4.8) \\
\end{array}$ \\
\hline Jamaica & $\begin{array}{c}12.5 \\
(7.1-21.2) \\
\end{array}$ & $\begin{array}{c}4.2 \\
(2.1-8.0) \\
\end{array}$ & $\begin{array}{c}17.7 \\
(9.9-25.5) \\
\end{array}$ & $\begin{array}{c}7.5 \\
(5.0-9.9) \\
\end{array}$ \\
\hline Mexico & $\begin{array}{c}41.3 \\
(36.0-46.8) \\
\end{array}$ & $\begin{array}{c}30.8 \\
(24.7-37.6) \\
\end{array}$ & $\begin{array}{c}37.6 \\
(30.2-45.0) \\
\end{array}$ & $\begin{array}{c}12.4 \\
(8.9-15.9) \\
\end{array}$ \\
\hline Panama & $\begin{array}{c}11.2 \\
(8.7-14.1) \\
\end{array}$ & $\begin{array}{c}11.1 \\
(9.2-13.5) \\
\end{array}$ & NA & NA \\
\hline Paraguay & $\begin{array}{c}25.3 \\
(23.2-27.7) \\
\end{array}$ & $\begin{array}{c}19.9 \\
(18.1-21.8) \\
\end{array}$ & $\begin{array}{c}33.2 \\
(29.4-37.0) \\
\end{array}$ & $\begin{array}{c}14.4 \\
(12.2-16.5) \\
\end{array}$ \\
\hline Peru & $\begin{array}{c}43.5 \\
(36.2-51.1) \\
\end{array}$ & $\begin{array}{c}22.2 \\
(16.7-28.8) \\
\end{array}$ & NA & NA \\
\hline Uruguay & $\begin{array}{c}31.3 \\
(29.5-33.2) \\
\end{array}$ & $\begin{array}{c}32.4 \\
(31.2-33.7) \\
\end{array}$ & $\begin{array}{c}36.6 \\
(32.2-41.0) \\
\end{array}$ & $\begin{array}{c}25.8 \\
(22.1-29.6) \\
\end{array}$ \\
\hline \multicolumn{5}{|c|}{ SOUTH-EAST ASIA REGION (SEAR) } \\
\hline Bangladesh & $\begin{array}{c}46.5 \\
(37.6-55.6) \\
\end{array}$ & $\begin{array}{c}4.4 \\
(1.2-14.1) \\
\end{array}$ & $\begin{array}{c}41.0 \\
(33.2-48.8) \\
\end{array}$ & $\begin{array}{c}0.7 \\
(0.3-1.1) \\
\end{array}$ \\
\hline India & $\begin{array}{c}17.7 \\
(14.5-21.3) \\
\end{array}$ & $\begin{array}{c}2 \\
(0.9-4.2) \\
\end{array}$ & $\begin{array}{c}25.8 \\
(20.8-30.8) \\
\end{array}$ & $\begin{array}{c}0.6 \\
(0.4-0.8) \\
\end{array}$ \\
\hline Indonesia & $\begin{array}{c}19.8 \\
(12.5-29.7) \\
\end{array}$ & $\begin{array}{c}2.3 \\
(1.2-4.5) \\
\end{array}$ & $\begin{array}{c}61.8 \\
(54.3-69.3) \\
\end{array}$ & $\begin{array}{c}3.7 \\
(3.3-4.2) \\
\end{array}$ \\
\hline Myanmar & $\begin{array}{c}24.7 \\
(23.5-26.1) \\
\end{array}$ & $\begin{array}{c}1.2 \\
(0.9-1.6) \\
\end{array}$ & $\begin{array}{c}42.5 \\
(37.0-47.9) \\
\end{array}$ & $\begin{array}{c}10.1 \\
(9.0-11.2) \\
\end{array}$ \\
\hline Nepal & $\begin{array}{c}34.5 \\
(13.1-64.7) \\
\end{array}$ & $\begin{array}{c}4.0 \\
(1.2-12.5) \\
\end{array}$ & $\begin{array}{c}25.2 \\
(20.4-30.0) \\
\end{array}$ & $\begin{array}{c}22.4 \\
(15.3-29.5)\end{array}$ \\
\hline Sri Lanka & $\begin{array}{c}8.6 \\
(7.0-10.7) \\
\end{array}$ & $\begin{array}{c}1.1 \\
(0.7-1.8) \\
\end{array}$ & $\begin{array}{c}24.4 \\
(19.6-29.1) \\
\end{array}$ & $\begin{array}{c}0.4 \\
(0.2-0.7) \\
\end{array}$ \\
\hline Thailand & $\begin{array}{c}1.0 \\
(0.5-2.1) \\
\end{array}$ & $\begin{array}{c}3.0 \\
(2.1-4.3) \\
\end{array}$ & $\begin{array}{c}37.3 \\
(32.9-41.8) \\
\end{array}$ & $\begin{array}{c}3.0 \\
(2.9-3.1) \\
\end{array}$ \\
\hline \multicolumn{5}{|c|}{ WESTERN PACIFIC REGION (WPR) } \\
\hline Cambodia & $\begin{array}{c}9.2 \\
(6.0-13.9) \\
\end{array}$ & 0.0 & $\begin{array}{c}30.3 \\
(26.5-34.1) \\
\end{array}$ & $\begin{array}{c}10.8 \\
(10.0-11.6) \\
\end{array}$ \\
\hline South Korea & $\begin{array}{c}22.5 \\
(18.1-27.5) \\
\end{array}$ & $\begin{array}{c}3.5 \\
(1.6-7.2) \\
\end{array}$ & $\begin{array}{c}53.8 \\
(51.4-56.1) \\
\end{array}$ & $\begin{array}{c}5.6 \\
(4.9-6.4) \\
\end{array}$ \\
\hline Viet Nam & $\begin{array}{c}20.8 \\
(19.7-21.9)\end{array}$ & $\begin{array}{c}2.6 \\
(2.3-3.0)\end{array}$ & $\begin{array}{c}42.0 \\
(36.4-47.6)\end{array}$ & $\begin{array}{c}1.9 \\
(1.2-2.5)\end{array}$ \\
\hline
\end{tabular}

NA Data not available. 
Table 3 Prevalence of Current Tobacco Use, by Sex, Region and Country, Medical Global Health Professions Student Survey 2005-2008

\begin{tabular}{|c|c|c|c|c|c|c|c|c|c|}
\hline \multirow[b]{2}{*}{$\begin{array}{l}\text { Country } \\
\text { (Site) }\end{array}$} & \multirow[b]{2}{*}{ Year } & \multicolumn{4}{|c|}{ Current cigarette smokers } & \multicolumn{4}{|c|}{ Current users of other tobacco products } \\
\hline & & $\begin{array}{l}\text { Total \% } \\
(95 \% \mathrm{Cl})\end{array}$ & $\begin{array}{l}\text { Male \% } \\
(95 \% \mathrm{CI})\end{array}$ & $\begin{array}{c}\text { Female \% } \\
(95 \% \mathrm{Cl})\end{array}$ & P-Value & $\begin{array}{l}\text { Total \% } \\
(95 \% \mathrm{CI})\end{array}$ & $\begin{array}{c}\text { Male \% } \\
(95 \% \mathrm{Cl})\end{array}$ & $\begin{array}{c}\text { Female } \% \\
(95 \% \mathrm{Cl})\end{array}$ & P-Value \\
\hline \multicolumn{10}{|c|}{ AFRICAN REGION(AFR) } \\
\hline Algeria & 2007 & $\begin{array}{c}9.0 \\
(8.1-9.9) \\
\end{array}$ & $\begin{array}{c}23.7 \\
(21.3-26.2) \\
\end{array}$ & $\begin{array}{c}3.0 \\
(2.5-3.7) \\
\end{array}$ & 0.0000 & $\begin{array}{c}4.1 \\
(3.6-4.7) \\
\end{array}$ & $\begin{array}{c}11.0 \\
(9.5-12.8) \\
\end{array}$ & $\begin{array}{c}1.1 \\
(0.8-1.5) \\
\end{array}$ & 0.0000 \\
\hline Kenya & 2008 & $\begin{array}{c}9.8 \\
(7.7-12.5)\end{array}$ & $\begin{array}{c}10.9 \\
(8.0-14.8)\end{array}$ & $\begin{array}{c}8.7 \\
(5.9-12.6)\end{array}$ & 0.3497 & $\begin{array}{c}4.9 \\
(3.5-7.0)\end{array}$ & $\begin{array}{c}5.1 \\
(3.2-8.1)\end{array}$ & $\begin{array}{c}4.9 \\
(2.9-8.1)\end{array}$ & 0.8937 \\
\hline Niger & 2008 & $\begin{array}{c}37.7 \\
(33.9-41.7)\end{array}$ & $\begin{array}{c}42.2 \\
(37.9-46.7)\end{array}$ & $\begin{array}{c}15.0 \\
(9.6-22.8)\end{array}$ & 0.0000 & $\begin{array}{c}27.7 \\
(22.0-34.3)\end{array}$ & $\begin{array}{c}41.9 \\
(31.4-53.3)\end{array}$ & $\begin{array}{c}18.6 \\
(12.6-26.8)\end{array}$ & 0.0006 \\
\hline Uganda & 2005 & $\begin{array}{c}2.8 \\
(1.8-4.2)\end{array}$ & $\begin{array}{c}4.1 \\
(2.7-6.3)\end{array}$ & 0.0 & 0.0000 & $\begin{array}{c}0.7 \\
(0.3-1.8)\end{array}$ & $\begin{array}{c}1.1 \\
(0.5-2.6) \\
\end{array}$ & 0.0 & 0.0225 \\
\hline \multicolumn{10}{|c|}{ EASTERN MEDITERRANEAN REGION (EMR) } \\
\hline Egypt & 2005 & $\begin{array}{c}7.9 \\
(5.7-10.7)\end{array}$ & $\begin{array}{c}12.9 \\
(9.9-16.5)\end{array}$ & $\begin{array}{c}1.2 \\
(0.5-3.0)\end{array}$ & 0.0000 & $\begin{array}{c}7.4 \\
(5.4-9.9)\end{array}$ & $\begin{array}{c}11.9 \\
(9.2-15.1)\end{array}$ & $\begin{array}{c}1.4 \\
(0.9-2.3)\end{array}$ & 0.0000 \\
\hline Gaza Strip/West Bank & 2007 & $\begin{array}{c}22.7 \\
(19.7-26.0) \\
\end{array}$ & $\begin{array}{c}39.1 \\
(33.8-44.6)\end{array}$ & $\begin{array}{c}9.4 \\
(6.8-12.8) \\
\end{array}$ & 0.0000 & $\begin{array}{c}12.3 \\
(10.0-14.9)\end{array}$ & $\begin{array}{c}24.1 \\
(19.7-29.0) \\
\end{array}$ & $\begin{array}{c}2.4 \\
(1.2-4.6)\end{array}$ & 0.0000 \\
\hline Iran & 2007 & $\begin{array}{c}5.6 \\
(4.6-6.9) \\
\end{array}$ & $\begin{array}{c}11.6 \\
(9.0-14.9) \\
\end{array}$ & $\begin{array}{c}2.8 \\
(1.9-4.0) \\
\end{array}$ & 0.0000 & $\begin{array}{c}9.9 \\
(8.5-11.6) \\
\end{array}$ & $\begin{array}{c}16.2 \\
(13.0-19.9) \\
\end{array}$ & $\begin{array}{c}7.0 \\
(5.5-8.8) \\
\end{array}$ & 0.0000 \\
\hline Iraq & 2005 & $\begin{array}{c}17.5 \\
(15.4-19.8) \\
\end{array}$ & $\begin{array}{c}20.4 \\
(17.2-24.1) \\
\end{array}$ & $\begin{array}{c}15.1 \\
(12.5-18.2) \\
\end{array}$ & 0.0201 & $\begin{array}{c}7.8 \\
(6.4-9.5) \\
\end{array}$ & $\begin{array}{c}4.7 \\
(3.2-6.9) \\
\end{array}$ & $\begin{array}{c}10.3 \\
(8.1-13.0) \\
\end{array}$ & 0.0003 \\
\hline Lebanon & 2006 & $\begin{array}{c}28.2 \\
(25.1-31.4)\end{array}$ & $\begin{array}{c}36.1 \\
(31.8-40.7)\end{array}$ & $\begin{array}{c}18.0 \\
(14.4-22.3)\end{array}$ & 0.0000 & $\begin{array}{c}21.8 \\
(19.2-24.8)\end{array}$ & $\begin{array}{c}21.7 \\
(18.2-25.8)\end{array}$ & $\begin{array}{c}21.9 \\
(18.1-26.4)\end{array}$ & 0.9449 \\
\hline Libyan Arab Jamahiriya & 2006 & $\begin{array}{c}10.1 \\
(9.1-11.2) \\
\end{array}$ & $\begin{array}{c}22.3 \\
(20.1-24.6) \\
\end{array}$ & $\begin{array}{c}1.9 \\
(1.4-2.7) \\
\end{array}$ & 0.0000 & $\begin{array}{c}8.3 \\
(7.4-9.3) \\
\end{array}$ & $\begin{array}{c}15.3 \\
(13.6-17.3) \\
\end{array}$ & $\begin{array}{c}3.6 \\
(2.8-4.5) \\
\end{array}$ & 0.0000 \\
\hline Saudi Arabia & 2006 & $\begin{array}{c}11.6 \\
(9.2-14.6) \\
\end{array}$ & $\begin{array}{c}13.1 \\
(10.0-16.9) \\
\end{array}$ & $\begin{array}{c}9.6 \\
(6.2-14.8) \\
\end{array}$ & 0.2156 & $\begin{array}{c}12.8 \\
(10.2-15.9) \\
\end{array}$ & $\begin{array}{c}13.9 \\
(10.7-17.7) \\
\end{array}$ & $\begin{array}{c}11.3 \\
(7.3-17.0)\end{array}$ & 0.3900 \\
\hline Sudan & 2007 & $\begin{array}{c}7.7 \\
(6.3-9.4) \\
\end{array}$ & $\begin{array}{c}19.6 \\
(15.9-23.9) \\
\end{array}$ & $\begin{array}{c}1.3 \\
(0.8-2.3) \\
\end{array}$ & 0.0000 & $\begin{array}{c}3.1 \\
(2.2-4.2) \\
\end{array}$ & $\begin{array}{c}8.0 \\
(5.8-10.9) \\
\end{array}$ & $\begin{array}{c}0.4 \\
(0.2-1.1)\end{array}$ & 0.0000 \\
\hline Syrian Arab Republic & 2006 & $\begin{array}{c}16.8 \\
(16.2-17.5)\end{array}$ & $\begin{array}{c}24.3 \\
(23.3-25.3)\end{array}$ & $\begin{array}{c}4.8 \\
(4.2-5.5)\end{array}$ & 0.0000 & $\begin{array}{c}23.6 \\
(22.8-24.4)\end{array}$ & $\begin{array}{c}30.6 \\
(29.6-31.7)\end{array}$ & $\begin{array}{c}12.1 \\
(11.2-13.2)\end{array}$ & 0.0000 \\
\hline Tunisia & 2007 & $\begin{array}{c}9.9 \\
(8.6-11.3)\end{array}$ & $\begin{array}{c}26.5 \\
(22.7-30.7)\end{array}$ & $\begin{array}{c}4.1 \\
(3.2-5.4)\end{array}$ & 0.0000 & $\begin{array}{c}7.4 \\
(6.3-8.7)\end{array}$ & $\begin{array}{c}20.6 \\
(17.2-24.5)\end{array}$ & $\begin{array}{c}3.0 \\
(2.2-4.1)\end{array}$ & 0.0000 \\
\hline \multicolumn{10}{|c|}{ EUROPEAN REGION (EUR) } \\
\hline Albania & 2005 & $\begin{array}{c}43.3 \\
(40.7-45.9) \\
\end{array}$ & $\begin{array}{c}65.1 \\
(59.8-69.9) \\
\end{array}$ & $\begin{array}{c}35.7 \\
(32.8-38.7) \\
\end{array}$ & 0.0043 & $\begin{array}{c}1.5 \\
(1.0-2.2) \\
\end{array}$ & $\begin{array}{c}3.1 \\
(1.8-5.4) \\
\end{array}$ & $\begin{array}{c}1.0 \\
(0.6-1.8) \\
\end{array}$ & 0.3508 \\
\hline Armenia & 2006 & $\begin{array}{c}20.4 \\
(16.1-25.4)\end{array}$ & $\begin{array}{c}52.5 \\
(41.7-63.0)\end{array}$ & $\begin{array}{c}8.3 \\
(5.2-13.1)\end{array}$ & 0.0000 & $\begin{array}{c}3.1 \\
(1.7-5.6)\end{array}$ & $\begin{array}{c}9.5 \\
(4.9-17.7)\end{array}$ & $\begin{array}{c}0.7 \\
(0.2-3.2)\end{array}$ & 0.0061 \\
\hline Bosnia \& Herzegovina & 2006 & $\begin{array}{c}40.3 \\
(39.2-41.5)\end{array}$ & $\begin{array}{c}45.0 \\
(43.0-47.0)\end{array}$ & $\begin{array}{c}37.8 \\
(36.4-39.2)\end{array}$ & 0.1689 & $\begin{array}{c}8.7 \\
(8.1-9.4) \\
\end{array}$ & $\begin{array}{c}10.9 \\
(9.7-12.2)\end{array}$ & $\begin{array}{c}7.5 \\
(6.8-8.3)\end{array}$ & 0.0417 \\
\hline Croatia & 2005 & $\begin{array}{c}36.6 \\
(34.1-39.2) \\
\end{array}$ & $\begin{array}{c}35.9 \\
(31.5-40.4) \\
\end{array}$ & $\begin{array}{c}37.1 \\
(34.1-40.3) \\
\end{array}$ & 0.6480 & $\begin{array}{c}10.7 \\
(9.2-12.4) \\
\end{array}$ & $\begin{array}{c}20.2 \\
(16.8-24.2) \\
\end{array}$ & $\begin{array}{c}6.3 \\
(5.0-8.0) \\
\end{array}$ & 0.0000 \\
\hline Czech Republic & 2006 & $\begin{array}{c}21.7 \\
(19.5-24.0) \\
\end{array}$ & $\begin{array}{c}26.2 \\
(21.9-30.9) \\
\end{array}$ & $\begin{array}{c}19.8 \\
(17.2-22.5) \\
\end{array}$ & 0.0158 & $\begin{array}{c}7.4 \\
(6.1-9.0) \\
\end{array}$ & $\begin{array}{c}11.1 \\
(8.3-14.6) \\
\end{array}$ & $\begin{array}{c}5.8 \\
(4.5-7.6) \\
\end{array}$ & 0.0035 \\
\hline Kyrgyzstan & 2008 & $\begin{array}{c}36.6 \\
(33.9-39.4) \\
\end{array}$ & $\begin{array}{c}50.0 \\
(45.5-54.5) \\
\end{array}$ & $\begin{array}{c}27.9 \\
(24.8-31.3) \\
\end{array}$ & 0.0000 & $\begin{array}{c}5.4 \\
(4.2-6.8) \\
\end{array}$ & $\begin{array}{c}11.4 \\
(8.8-14.5) \\
\end{array}$ & $\begin{array}{c}1.5 \\
(0.8-2.6) \\
\end{array}$ & 0.0000 \\
\hline Lithuania & 2006 & $\begin{array}{c}27.3 \\
(23.5-31.4) \\
\end{array}$ & $\begin{array}{c}48.6 \\
(39.7-57.7) \\
\end{array}$ & $\begin{array}{c}19.5 \\
(15.8-23.8) \\
\end{array}$ & 0.0000 & $\begin{array}{c}15.1 \\
(12.3-18.5) \\
\end{array}$ & $\begin{array}{c}33.2 \\
(25.4-42.1) \\
\end{array}$ & $\begin{array}{c}8.2 \\
(6.0-11.2) \\
\end{array}$ & 0.0000 \\
\hline Russian Federation & 2006 & $\begin{array}{c}38.8 \\
(37.6-39.9)\end{array}$ & $\begin{array}{c}46.1 \\
(44.2-48.1)\end{array}$ & $\begin{array}{c}34.9 \\
(33.5-36.2)\end{array}$ & 0.0000 & $\begin{array}{c}10.9 \\
(10.2-11.6)\end{array}$ & $\begin{array}{c}19.3 \\
(17.8-20.9)\end{array}$ & $\begin{array}{c}6.5 \\
(5.8-7.2)\end{array}$ & 0.0000 \\
\hline Serbia & 2006 & $\begin{array}{c}34.7 \\
(33.2-36.2) \\
\end{array}$ & $\begin{array}{c}31.2 \\
(28.7-33.8) \\
\end{array}$ & $\begin{array}{c}36.7 \\
(34.9-38.6) \\
\end{array}$ & 0.0007 & $\begin{array}{c}18.0 \\
(16.8-19.2) \\
\end{array}$ & $\begin{array}{c}17.7 \\
(15.7-19.8) \\
\end{array}$ & $\begin{array}{c}18.4 \\
(17.0-20.0) \\
\end{array}$ & 0.5587 \\
\hline Slovakia & 2006 & $\begin{array}{c}30.4 \\
(29.0-31.9) \\
\end{array}$ & $\begin{array}{c}36.5 \\
(33.6-39.4) \\
\end{array}$ & $\begin{array}{c}27.9 \\
(26.2-29.6)\end{array}$ & 0.0000 & $\begin{array}{c}6.4 \\
(5.7-7.2) \\
\end{array}$ & $\begin{array}{c}8.6 \\
(7.1-10.5) \\
\end{array}$ & $\begin{array}{c}5.6 \\
(4.8-6.5) \\
\end{array}$ & 0.0017 \\
\hline Slovenia & 2007 & $\begin{array}{c}20.9 \\
(17.3-25.0)\end{array}$ & $\begin{array}{c}22.5 \\
(15.9-30.8)\end{array}$ & $\begin{array}{c}20.2 \\
(16.0-25.1)\end{array}$ & 0.6020 & $\begin{array}{c}5.6 \\
(3.8-8.2)\end{array}$ & $\begin{array}{c}14.3 \\
(9.2-21.5)\end{array}$ & $\begin{array}{c}2.0 \\
(0.9-4.4)\end{array}$ & 0.0002 \\
\hline
\end{tabular}


Table 3 Prevalence of Current Tobacco Use, by Sex, Region and Country, Medical Global Health Professions Student Survey 2005-2008 (Continued)

\begin{tabular}{|c|c|c|c|c|c|c|c|c|c|}
\hline \multicolumn{10}{|c|}{ REGION OF THE AMERICAS (AMR) } \\
\hline Argentina & 2005 & $\begin{array}{c}35.5 \\
(33.6-37.4)\end{array}$ & $\begin{array}{c}33.4 \\
(30.4-36.4)\end{array}$ & $\begin{array}{c}36.5 \\
(34.1-39.1)\end{array}$ & 0.1140 & $\begin{array}{c}6.4 \\
(5.4-7.4)\end{array}$ & $\begin{array}{c}9.2 \\
(7.5-11.3)\end{array}$ & $\begin{array}{c}4.5 \\
(3.5-5.7)\end{array}$ & 0.0000 \\
\hline Bolivia & 2006 & $\begin{array}{c}41.1 \\
(35.3-47.2)\end{array}$ & $\begin{array}{c}50.3 \\
(43.9-56.7)\end{array}$ & $\begin{array}{c}31.7 \\
(24.9-39.4)\end{array}$ & 0.0017 & $\begin{array}{c}10.6 \\
(8.7-12.8)\end{array}$ & $\begin{array}{c}14.3 \\
(11.1-18.4)\end{array}$ & $\begin{array}{c}6.7 \\
(4.9-9.0)\end{array}$ & 0.0075 \\
\hline Brazil (Rio de Janeiro) & 2006 & $\begin{array}{c}16.9 \\
(15.6-18.2) \\
\end{array}$ & $\begin{array}{c}19.5 \\
(17.5-21.6) \\
\end{array}$ & $\begin{array}{c}14.6 \\
(13.1-16.3) \\
\end{array}$ & 0.0002 & $\begin{array}{c}5.2 \\
(4.5-6.0) \\
\end{array}$ & $\begin{array}{c}7.1 \\
(5.9-8.5) \\
\end{array}$ & $\begin{array}{c}3.4 \\
(2.7-4.3)\end{array}$ & 0.0000 \\
\hline Chile & 2008 & $\begin{array}{c}28.4 \\
(27.1-29.6)\end{array}$ & $\begin{array}{c}27.1 \\
(25.5-28.9)\end{array}$ & $\begin{array}{c}29.3 \\
(27.5-31.3)\end{array}$ & 0.0878 & $\begin{array}{c}7.6 \\
(6.8-8.4)\end{array}$ & $\begin{array}{c}9.3 \\
(8.2-10.5)\end{array}$ & $\begin{array}{c}5.5 \\
(4.6-6.5)\end{array}$ & 0.0000 \\
\hline Costa Rica & 2006 & 32.8 & 38.6 & 29.3 & * & 13.7 & 19.2 & 10.7 & * \\
\hline Cuba (Havana) & 2008 & $\begin{array}{c}29.5 \\
(27.6-31.4)\end{array}$ & $\begin{array}{c}41.1 \\
(38.0-44.3)\end{array}$ & $\begin{array}{c}21.2 \\
(19.1-23.5)\end{array}$ & 0.0000 & $\begin{array}{c}4.1 \\
(3.4-5.0)\end{array}$ & $\begin{array}{c}4.1 \\
(3.1-5.5)\end{array}$ & $\begin{array}{c}4.1 \\
(3.2-5.3)\end{array}$ & 0.9874 \\
\hline Guatemala & 2008 & $\begin{array}{c}22.5 \\
(19.8-25.4)\end{array}$ & $\begin{array}{c}27.5 \\
(23.4-32.1)\end{array}$ & $\begin{array}{c}17.8 \\
(14.6-21.6)\end{array}$ & 0.0007 & $\begin{array}{c}13.5 \\
(11.4-15.9)\end{array}$ & $\begin{array}{c}17.4 \\
(14.0-21.4)\end{array}$ & $\begin{array}{c}10.3 \\
(7.8-13.3)\end{array}$ & 0.0024 \\
\hline Jamaica & 2008 & $\begin{array}{c}6.7 \\
(4.3-10.3)\end{array}$ & $\begin{array}{c}12.5 \\
(7.1-21.2)\end{array}$ & $\begin{array}{c}4.2 \\
(2.1-8.0)\end{array}$ & 0.0286 & $\begin{array}{c}3.8 \\
(2.1-6.7)\end{array}$ & $\begin{array}{c}12.5 \\
(7.1-21.2)\end{array}$ & 0.0 & 0.0005 \\
\hline Mexico & 2006 & $\begin{array}{c}35.3 \\
(29.8-41.3)\end{array}$ & $\begin{array}{c}41.3 \\
(36.0-46.8)\end{array}$ & $\begin{array}{c}30.8 \\
(24.7-37.6)\end{array}$ & 0.0010 & $\begin{array}{c}4.5 \\
(3.3-6.3)\end{array}$ & $\begin{array}{c}6.3 \\
(4.5-8.8) \\
\end{array}$ & $\begin{array}{c}2.7 \\
(2.0-3.8)\end{array}$ & 0.0027 \\
\hline Panama & 2008 & $\begin{array}{c}11.1 \\
(9.6-12.9) \\
\end{array}$ & $\begin{array}{c}11.2 \\
(8.7-14.1) \\
\end{array}$ & $\begin{array}{c}11.1 \\
(9.2-13.5) \\
\end{array}$ & 0.9956 & $\begin{array}{c}2.9 \\
(2.1-3.9) \\
\end{array}$ & $\begin{array}{c}3.6 \\
(2.3-5.6) \\
\end{array}$ & $\begin{array}{c}1.9 \\
(1.1-3.1) \\
\end{array}$ & 0.0578 \\
\hline Paraguay & 2008 & $\begin{array}{c}22.3 \\
(20.9-23.8) \\
\end{array}$ & $\begin{array}{c}25.3 \\
(23.2-27.7) \\
\end{array}$ & $\begin{array}{c}19.9 \\
(18.1-21.8) \\
\end{array}$ & 0.0003 & $\begin{array}{c}6.8 \\
(6.0-7.7) \\
\end{array}$ & $\begin{array}{c}8.3 \\
(7.0-9.8) \\
\end{array}$ & $\begin{array}{c}5.6 \\
(4.6-6.8) \\
\end{array}$ & 0.0029 \\
\hline Peru & 2006 & $\begin{array}{c}32.7 \\
(28.5-37.3) \\
\end{array}$ & $\begin{array}{c}43.5 \\
(36.2-51.1) \\
\end{array}$ & $\begin{array}{c}22.2 \\
(16.7-28.8) \\
\end{array}$ & 0.0007 & $\begin{array}{c}6.4 \\
(3.4-11.8) \\
\end{array}$ & $\begin{array}{c}9.0 \\
(5.2-14.9) \\
\end{array}$ & $\begin{array}{c}3.6 \\
(1.4-9.1) \\
\end{array}$ & 0.0003 \\
\hline Uruguay & 2008 & $\begin{array}{c}32.3 \\
(31.2-33.3)\end{array}$ & $\begin{array}{c}31.3 \\
(29.5-33.2)\end{array}$ & $\begin{array}{c}32.4 \\
(31.2-33.7)\end{array}$ & 0.3222 & $\begin{array}{c}5.8 \\
(5.3-6.3)\end{array}$ & $\begin{array}{c}12.5 \\
(11.3-13.9)\end{array}$ & $\begin{array}{c}2.6 \\
(2.2-3.0)\end{array}$ & 0.0000 \\
\hline \multicolumn{10}{|c|}{ SOUTH-EAST ASIA REGION (SEAR) } \\
\hline Bangladesh & 2006 & $\begin{array}{c}27.2 \\
(20.8-34.8) \\
\end{array}$ & $\begin{array}{c}46.5 \\
(37.6-55.6) \\
\end{array}$ & $\begin{array}{c}4.4 \\
(1.2-14.1) \\
\end{array}$ & 0.0000 & $\begin{array}{c}11.9 \\
(4.3-28.7) \\
\end{array}$ & $\begin{array}{c}13.3 \\
(3.7-38.2) \\
\end{array}$ & $\begin{array}{c}9.9 \\
(4.6-20.0) \\
\end{array}$ & 0.5038 \\
\hline India & 2005 & $\begin{array}{c}11.6 \\
(8.8-15.2)\end{array}$ & $\begin{array}{c}17.7 \\
(14.5-21.3)\end{array}$ & $\begin{array}{c}2.0 \\
(0.9-4.2)\end{array}$ & 0.0000 & $\begin{array}{c}5.4 \\
(3.5-8.3)\end{array}$ & $\begin{array}{c}7.5 \\
(5.2-10.6)\end{array}$ & $\begin{array}{c}2.2 \\
(0.8-6.4)\end{array}$ & 0.0028 \\
\hline Indonesia & 2006 & $\begin{array}{c}8.6 \\
(5.4-13.5)\end{array}$ & $\begin{array}{c}19.8 \\
(12.5-29.7)\end{array}$ & $\begin{array}{c}2.3 \\
(1.2-4.5)\end{array}$ & 0.0024 & $\begin{array}{c}0.9 \\
(0.4-2.2)\end{array}$ & $\begin{array}{c}2.2 \\
(0.9-5.0)\end{array}$ & $\begin{array}{c}0.1 \\
(0.0-1.1)\end{array}$ & 0.0274 \\
\hline Myanmar & 2006 & $\begin{array}{c}12.4 \\
(11.7-13.1)\end{array}$ & $\begin{array}{c}24.7 \\
(23.5-26.1)\end{array}$ & $\begin{array}{c}1.2 \\
(0.9-1.6)\end{array}$ & 0.0000 & $\begin{array}{c}11.0 \\
(10.3-11.6)\end{array}$ & $\begin{array}{c}19.8 \\
(18.6-21.0) \\
\end{array}$ & $\begin{array}{c}2.7 \\
(2.3-3.2)\end{array}$ & 0.0000 \\
\hline Nepal & 2005 & $\begin{array}{c}23.5 \\
(9.1-48.5)\end{array}$ & $\begin{array}{c}34.5 \\
(13.1-64.7)\end{array}$ & $\begin{array}{c}4.0 \\
(1.2-12.5)\end{array}$ & 0.0372 & $\begin{array}{c}14.4 \\
(6.7-28.1)\end{array}$ & $\begin{array}{c}19.3 \\
(6.8-43.6)\end{array}$ & $\begin{array}{c}5.4 \\
(1.0-23.9)\end{array}$ & 0.1694 \\
\hline Sri Lanka & 2006 & $\begin{array}{c}4.1 \\
(3.4-5.0)\end{array}$ & $\begin{array}{c}8.6 \\
(7.0-10.7)\end{array}$ & $\begin{array}{c}1.1 \\
(0.7-1.8)\end{array}$ & 0.0000 & $\begin{array}{c}3.6 \\
(2.9-4.4)\end{array}$ & $\begin{array}{c}6.8 \\
(5.4-8.6)\end{array}$ & $\begin{array}{c}1.4 \\
(0.9-2.1)\end{array}$ & 0.0000 \\
\hline Thailand & 2006 & $\begin{array}{c}2.1 \\
(1.6-2.9)\end{array}$ & $\begin{array}{c}1.0 \\
(0.5-2.1)\end{array}$ & $\begin{array}{c}3.0 \\
(2.1-4.3)\end{array}$ & 0.0029 & $\begin{array}{c}1.7 \\
(1.1-2.5)\end{array}$ & $\begin{array}{c}2.3 \\
(1.4-3.9)\end{array}$ & $\begin{array}{c}0.9 \\
(0.5-1.7)\end{array}$ & 0.0443 \\
\hline \multicolumn{10}{|c|}{ WESTERN PACIFIC REGION (WPR) } \\
\hline Cambodia & 2005 & $\begin{array}{c}6.4 \\
(4.1-9.7)\end{array}$ & $\begin{array}{c}9.2 \\
(6.0-13.9)\end{array}$ & 0.0 & 0.0000 & $\begin{array}{c}3.6 \\
(2.0-6.4)\end{array}$ & $\begin{array}{c}5.3 \\
(3.0-9.2)\end{array}$ & 0.0 & 0.0007 \\
\hline South Korea & 2006 & $\begin{array}{c}16.0 \\
(12.0-21.1)\end{array}$ & $\begin{array}{c}22.5 \\
(18.1-27.5)\end{array}$ & $\begin{array}{c}3.5 \\
(1.6-7.2)\end{array}$ & 0.0000 & $\begin{array}{c}4.8 \\
(3.0-7.4)\end{array}$ & $\begin{array}{c}6.9 \\
(4.7-10.0)\end{array}$ & $\begin{array}{c}1.0 \\
(0.5-2.1)\end{array}$ & 0.0001 \\
\hline Viet Nam & 2007 & $\begin{array}{c}11.2 \\
(10.6-11.7)\end{array}$ & $\begin{array}{c}20.8 \\
(19.7-21.9)\end{array}$ & $\begin{array}{c}2.6 \\
(2.3-3.0)\end{array}$ & 0.0000 & $\begin{array}{c}3.3 \\
(3.0-3.6)\end{array}$ & $\begin{array}{c}5.0 \\
(4.4-5.6)\end{array}$ & $\begin{array}{c}1.7 \\
(1.4-2.0)\end{array}$ & 0.0000 \\
\hline
\end{tabular}

\footnotetext{
* Census and $100 \%$ school, class and student response rates.
} 
Data presented in Table 2 compared GHPSS medical current cigarette smoking prevalence with adult cigarette prevalence data compiled from the WHO Report on the Global Tobacco Epidemic (GTCR), 2008 (WHO, 2008). Of the 48 sites included that conducted the medical GHPSS, 41 sites reported adult cigarette prevalence data in the GTCR 2008 report. Among males there was no difference in cigarette prevalence in 20 sites, while in 18 sites the adult prevalence was higher than the GHPSS medical student data, and in three of the sites medical students had higher cigarette smoking rates compared to the adult population. For females, 17 sites reported no difference between the medical students and adult cigarette smoking prevalence rates, while in four sites the adult cigarettes smoking prevalence was higher than medical students, and in 19 sites the medical students had higher rates of cigarettes smoking prevalence compared to the adult population.

\section{Desire for Cessation}

Over $60 \%$ of the students who reported that they were current cigarette smokers expressed a desire to quit smoking cigarettes in 26 of the 48 sites (Table 4 ). In 4 of the 48 sites less than half of the student who reported that they were current cigarette smokers expressed a desire to quit smoking cigarettes - only a quarter of the students in Bangladesh reported a desire to quit smoking cigarettes. Desire for current cigarettes smokers to quit smoking was over $55 \%$ in all of AFR sites, in 7 of the 10 sites in EMR, in 9 of 11 sites in EUR, in 10 of 13 sites in AMR, in 2 of 3 sites in WPR, and in 6 of the 7 sites in the SEAR region.

\section{Exposure to Secondhand Smoke (SHS)}

Over $50 \%$ of the students reported that they had been exposed to SHS in their home in the past 7 days in 15 of the 48 sites (Table 4). Over 70\% reported exposure to SHS at home in Albania and Cuba. Less than 50\% of the students in all 4 AFR sites reported exposure to SHS at home in the past 7 days; whereas exposure at home was greater than $50 \%$ in 4 of 10 sites in EMR, in 6 of 11 sites in EUR, in 2 of 13 sites in AMR, in 2 of 3 sites in WPR and in Myanmar in SEAR sites.

Over $70 \%$ of the students reported that they had been exposed to SHS in public places in the past 7 days in 29 of the 48 sites (Table 4). Exposure to SHS in public places was greater than $60 \%$ in 3 of 4 sites in AFR and in all 3 WPR sites; greater than $70 \%$ in 3 of 10 sites in EMR and in 9 of 13 sites in AMR and greater than $80 \%$ in 8 of 11 sites in EUR (Table 4).

The proportion of students reporting their schools have an official policy banning smoking in school buildings and clinics was over $60 \%$ in 21 of the 48 sites; and less than $20 \%$ in 6 sites (Table 4). Having a policy was least likely in EMR and most likely in EUR and WPR. Over $70 \%$ of the students reported enforcement of the policy in 15 of the 48 sites. Enforcement was less than $30 \%$ in Algeria, Gaza Strip/West Bank, Brazil, and Guatemala.

\section{Health Professional Roles and Training}

Over $80 \%$ of the students thought health professionals have a role in giving advice about smoking cessation to patients in 42 of 46 sites, with 30 over $90 \%$ (Table 5). The lowest was in Slovakia (59.7\%). Over $80 \%$ of the students thought health professionals should get specific training on cessation techniques in 41 of the 48 sites, with 33 over $90 \%$. The lowest was in Czech Republic (60.8\%). Less than $40 \%$ of the students reported having ever received some kind of formal training in their professional school on cessation approaches to use with their patients in 46 of the 48 sites; less than $20 \%$ in 16 sites, and less than $10 \%$ in 6 sites. Over $40 \%$ of the students had received formal training in Niger (46.4\%) and Myanmar (43.7\%).

\section{Discussion \\ Tobacco Use}

Findings from the Medical GHPSS show that over 20\% of medical students currently smoke cigarettes in 26 of 48 sites and over $40 \%$ in 3 sites. Use of other forms of tobacco was over $10 \%$ in 15 of 48 sites and over $20 \%$ in 2 sites. Not only does tobacco use endanger the health of medical students, but it is also known to negatively influence the health professionals to deliver effective anti-tobacco counseling when they start seeing patients [13]. Physicians who have healthy personal habits are more likely to discuss related preventive behaviors with their patients [14]. Thus the current smoking prevalence rates for medical students are disconcerting given that among the 41 sites that contained adult smoking prevalence data; over $55 \%$ reported no difference between medical students and the general adult population or the medical students had higher rates. For males, in 23 of the $41(56.1 \%)$ sites there was no difference or male medical students had higher rates of smoking compared to the general adult male population. While among females, female medical students had higher rates of cigarette smoking prevalence compared to the general adult female population in 36 of the 40 (90\%) countries, or there was no difference.

Since physician counseling on smoking cessation can have a positive effect on patient's smoking habits health promotion programs including tobacco cessation services for medical students may play a significant role in the future delivery of patient cessation counseling practices [15]. Therefore educational institutions training medical students should help their students quit using 
Table 4 Exposure to Secondhand Smoke (At Home and in Public Places) and School Policy and Enforcement Regarding Bans on Smoking, by Region and Country, Medical Global Health Professions Student Survey, 2005-2008

\begin{tabular}{|c|c|c|c|c|c|c|}
\hline & & $\begin{array}{l}\text { In the past } 7 \text { days, } \\
\text { had someone smoke } \\
\text { in their presence } \\
\text { and their home }\end{array}$ & $\begin{array}{l}\text { In the past } 7 \text { days, had } \\
\text { someone smoke in } \\
\text { their presence other } \\
\text { than in their home }\end{array}$ & $\begin{array}{l}\text { Had an official } \\
\text { policy banning } \\
\text { smoking in school } \\
\text { buildings and } \\
\text { clinics }\end{array}$ & $\begin{array}{l}\text { Had an official policy } \\
\text { banning smoking in school } \\
\text { buildings and clinics and } \\
\text { the policy was enforced }\end{array}$ & $\begin{array}{l}\text { Current cigarette } \\
\text { smokers who want } \\
\text { to stop smoking } \\
\text { cigarettes now }\end{array}$ \\
\hline $\begin{array}{l}\text { Country } \\
\text { (Site) }\end{array}$ & Year & $\begin{array}{l}\text { Total \% } \\
(95 \% \mathrm{Cl}) \\
\end{array}$ & $\begin{array}{c}\text { Total \% } \\
(95 \% \mathrm{Cl}) \\
\end{array}$ & $\begin{array}{c}\text { Total \% } \\
(95 \% \mathrm{Cl}) \\
\end{array}$ & $\begin{array}{c}\text { Total \% } \\
(95 \% \mathrm{Cl}) \\
\end{array}$ & $\begin{array}{c}\text { Total \% } \\
(95 \% \mathrm{Cl}) \\
\end{array}$ \\
\hline \multicolumn{7}{|c|}{ AFRICAN REGION (AFR) } \\
\hline Algeria & 2007 & $\begin{array}{c}27.0 \\
(25.7-28.3)\end{array}$ & $\begin{array}{c}38.4 \\
(37.0-39.8)\end{array}$ & $\begin{array}{c}29.8 \\
(28.5-31.1)\end{array}$ & $\begin{array}{c}25.0 \\
(22.7-27.4)\end{array}$ & $\begin{array}{c}68.7 \\
(63.1-73.7)\end{array}$ \\
\hline Kenya & 2008 & $\begin{array}{c}43.9 \\
(40.1-47.9) \\
\end{array}$ & $\begin{array}{c}64.5 \\
(60.6-68.1) \\
\end{array}$ & $\begin{array}{c}60.1 \\
(55.7-64.3) \\
\end{array}$ & $\begin{array}{c}44.4 \\
(37.4-51.6) \\
\end{array}$ & $\begin{array}{c}58.3 \\
(43.8-71.5)\end{array}$ \\
\hline Niger & 2008 & $\begin{array}{c}31.7 \\
(30.1-33.3) \\
\end{array}$ & $\begin{array}{c}71.2 \\
(69.6-72.7) \\
\end{array}$ & $\begin{array}{c}44.6 \\
(42.9-46.3) \\
\end{array}$ & $\begin{array}{c}42.8 \\
(40.3-45.3) \\
\end{array}$ & $\begin{array}{c}93.6 \\
(89.4-96.3) \\
\end{array}$ \\
\hline Uganda & 2005 & $\begin{array}{c}38.1 \\
(34.7-41.5)\end{array}$ & $\begin{array}{c}61.1 \\
(57.6-64.5)\end{array}$ & $\begin{array}{c}22.3 \\
(18.9-26.2)\end{array}$ & $\begin{array}{c}40.5 \\
(31.9-49.8)\end{array}$ & * \\
\hline \multicolumn{7}{|c|}{ EASTERN MEDITERRANEAN REGION (EMR) } \\
\hline Egypt & 2005 & $\begin{array}{c}45.6 \\
(41.7-49.5)\end{array}$ & $\begin{array}{c}78.4 \\
(76.1-80.6)\end{array}$ & $\begin{array}{c}31.1 \\
(26.2-36.5)\end{array}$ & $\begin{array}{c}41.1 \\
(32.1-50.8)\end{array}$ & $\begin{array}{c}66.0 \\
(47.0-81.0)\end{array}$ \\
\hline $\begin{array}{l}\text { Gaza Strip/ } \\
\text { West Bank }\end{array}$ & 2007 & $\begin{array}{c}63.3 \\
(59.7-66.8)\end{array}$ & $\begin{array}{c}67.1 \\
(63.6-70.5)\end{array}$ & $\begin{array}{c}30.0 \\
(26.7-33.5)\end{array}$ & $\begin{array}{c}18.8 \\
(13.8-25.1)\end{array}$ & * \\
\hline Iran & 2007 & $\begin{array}{c}31.2 \\
(28.8-33.7) \\
\end{array}$ & $\begin{array}{c}54.1 \\
(51.5-56.7) \\
\end{array}$ & $\begin{array}{c}33.5 \\
(31.1-36.1) \\
\end{array}$ & $\begin{array}{c}66.9 \\
(62.5-71.0) \\
\end{array}$ & $\begin{array}{c}33.5 \\
(21.7-47.9) \\
\end{array}$ \\
\hline Iraq & 2005 & $\begin{array}{c}50.2 \\
(47.4-53.1) \\
\end{array}$ & $\begin{array}{c}60.6 \\
(57.8-63.4) \\
\end{array}$ & $\begin{array}{c}9.4 \\
(7.9-11.2) \\
\end{array}$ & $\begin{array}{c}30.5 \\
(22.1-40.6) \\
\end{array}$ & $\begin{array}{c}70.2 \\
(56.1-81.4) \\
\end{array}$ \\
\hline Lebanon & 2006 & $\begin{array}{c}54.7 \\
(51.3-58.1) \\
\end{array}$ & $\begin{array}{c}80.7 \\
(77.9-83.3) \\
\end{array}$ & $\begin{array}{c}51.6 \\
(48.2-55.0) \\
\end{array}$ & $\begin{array}{c}61.9 \\
(57.2-66.4) \\
\end{array}$ & $\begin{array}{c}40.4 \\
(32.1-49.3) \\
\end{array}$ \\
\hline $\begin{array}{l}\text { Libyan Arab } \\
\text { Jamahiriya }\end{array}$ & 2006 & $\begin{array}{c}45.6 \\
(43.9-47.2) \\
\end{array}$ & $\begin{array}{c}59.6 \\
(58.0-61.2) \\
\end{array}$ & $\begin{array}{c}11.5 \\
(10.5-12.6) \\
\end{array}$ & $\begin{array}{c}44.2 \\
(38.8-49.7) \\
\end{array}$ & $\begin{array}{c}72.2 \\
(65.6-78.0)\end{array}$ \\
\hline $\begin{array}{l}\text { Saudi } \\
\text { Arabia }\end{array}$ & 2006 & $\begin{array}{c}31.0 \\
(27.3-34.9) \\
\end{array}$ & $\begin{array}{c}60.9 \\
(57.0-64.7) \\
\end{array}$ & $\begin{array}{c}59.8 \\
(55.8-63.7) \\
\end{array}$ & $\begin{array}{c}75.7 \\
(70.4-80.3) \\
\end{array}$ & $\begin{array}{c}75.9 \\
(61.9-86.0) \\
\end{array}$ \\
\hline Sudan & 2007 & $\begin{array}{c}40.6 \\
(37.7-43.5)\end{array}$ & $\begin{array}{c}68.5 \\
(65.6-71.2)\end{array}$ & $\begin{array}{c}18.3 \\
(16.2-20.7)\end{array}$ & $\begin{array}{c}68.3 \\
(61.6-74.4)\end{array}$ & $\begin{array}{c}92.1 \\
(80.7-97.0)\end{array}$ \\
\hline $\begin{array}{l}\text { Syrian Arab } \\
\text { Republic }\end{array}$ & 2006 & $\begin{array}{c}59.9 \\
(59.0-60.8) \\
\end{array}$ & $\begin{array}{c}79.3 \\
(78.5-80.0) \\
\end{array}$ & $\begin{array}{c}10.2 \\
(9.7-10.8) \\
\end{array}$ & $\begin{array}{c}48.6 \\
(45.8-51.5) \\
\end{array}$ & $\begin{array}{c}58.8 \\
(56.2-61.4) \\
\end{array}$ \\
\hline Tunisia & 2007 & $\begin{array}{c}44.5 \\
(42.1-47.0) \\
\end{array}$ & $\begin{array}{c}65.2 \\
(62.9-67.5) \\
\end{array}$ & $\begin{array}{c}36.6 \\
(34.3-39.1) \\
\end{array}$ & $\begin{array}{c}35.4 \\
(31.5-39.5) \\
\end{array}$ & $\begin{array}{c}61.8 \\
(51.9-70.8) \\
\end{array}$ \\
\hline \multicolumn{7}{|c|}{ EUROPEAN REGION (EUR) } \\
\hline Albania & 2005 & $\begin{array}{c}72.5 \\
(70.3-74.7) \\
\end{array}$ & $\begin{array}{c}95.8 \\
(94.7-96.7) \\
\end{array}$ & $\begin{array}{c}14.1 \\
(12.1-16.4) \\
\end{array}$ & $\begin{array}{c}41.4 \\
(33.7-49.6) \\
\end{array}$ & $\begin{array}{c}90.1 \\
(85.7-93.3) \\
\end{array}$ \\
\hline Armenia & 2006 & $\begin{array}{c}65.1 \\
(59.5-70.4) \\
\end{array}$ & $\begin{array}{c}80.0 \\
(74.9-84.2) \\
\end{array}$ & $\begin{array}{c}54.0 \\
(48.1-59.8) \\
\end{array}$ & $\begin{array}{c}90.5 \\
(84.8-94.2) \\
\end{array}$ & $\begin{array}{c}71.3 \\
(50.2-86.0) \\
\end{array}$ \\
\hline $\begin{array}{l}\text { Bosnia \& } \\
\text { Herzegovina }\end{array}$ & 2006 & $\begin{array}{c}53.0 \\
(51.8-54.2) \\
\end{array}$ & $\begin{array}{c}91.1 \\
(90.4-91.8) \\
\end{array}$ & $\begin{array}{c}58.8 \\
(57.6-59.9) \\
\end{array}$ & $\begin{array}{c}30.1 \\
(28.7-31.6) \\
\end{array}$ & $\begin{array}{c}46.6 \\
(44.4-48.9) \\
\end{array}$ \\
\hline Croatia & 2005 & $\begin{array}{c}50.4 \\
(47.9-53.0) \\
\end{array}$ & $\begin{array}{c}95.2 \\
(93.9-96.2) \\
\end{array}$ & $\begin{array}{c}83.1 \\
(80.7-85.2) \\
\end{array}$ & $\begin{array}{c}73.8 \\
(70.8-76.7) \\
\end{array}$ & $\begin{array}{c}55.2 \\
(50.0-60.3)\end{array}$ \\
\hline $\begin{array}{l}\text { Czech } \\
\text { Republic } \\
\end{array}$ & 2006 & $\begin{array}{c}25.9 \\
(23.6-28.4) \\
\end{array}$ & $\begin{array}{c}84.9 \\
(82.8-86.7) \\
\end{array}$ & $\begin{array}{c}95.7 \\
(94.4-96.7) \\
\end{array}$ & $\begin{array}{c}51.9 \\
(49.0-54.8) \\
\end{array}$ & $\begin{array}{c}55.2 \\
(47.7-62.6)\end{array}$ \\
\hline Kyrgyzstan & 2008 & $\begin{array}{c}41.1 \\
(38.3-43.9) \\
\end{array}$ & $\begin{array}{c}82.1 \\
(79.9-84.2) \\
\end{array}$ & $\begin{array}{c}50.5 \\
(47.6-53.3) \\
\end{array}$ & $\begin{array}{c}70.2 \\
(66.1-74.0) \\
\end{array}$ & $\begin{array}{c}84.0 \\
(79.1-87.9) \\
\end{array}$ \\
\hline Lithuania & 2006 & $\begin{array}{c}34.9 \\
(30.7-39.3) \\
\end{array}$ & $\begin{array}{c}68.1 \\
(63.9-72.1) \\
\end{array}$ & $\begin{array}{c}78.5 \\
(74.3-82.2) \\
\end{array}$ & $\begin{array}{c}57.3 \\
(52.3-62.2) \\
\end{array}$ & $\begin{array}{c}60.9 \\
(49.2-71.4) \\
\end{array}$ \\
\hline $\begin{array}{l}\text { Russian } \\
\text { Federation } \\
\end{array}$ & 2006 & $\begin{array}{c}48.8 \\
(47.7-49.9) \\
\end{array}$ & $\begin{array}{c}85.4 \\
(84.6-86.2) \\
\end{array}$ & $\begin{array}{c}74.6 \\
(73.6-75.6) \\
\end{array}$ & $\begin{array}{c}34.2 \\
(33.0-35.5) \\
\end{array}$ & $\begin{array}{c}68.2 \\
(66.2-70.2) \\
\end{array}$ \\
\hline Serbia & 2006 & $\begin{array}{c}67.8 \\
(66.3-69.2)\end{array}$ & $\begin{array}{c}89.5 \\
(88.4-90.4)\end{array}$ & $\begin{array}{c}67.4 \\
(65.9-68.9)\end{array}$ & $\begin{array}{c}81.2 \\
(79.6-82.6)\end{array}$ & $\begin{array}{c}57.7 \\
(54.7-60.8)\end{array}$ \\
\hline
\end{tabular}


Table 4 Exposure to Secondhand Smoke (At Home and in Public Places) and School Policy and Enforcement Regarding Bans on Smoking, by Region and Country, Medical Global Health Professions Student Survey, 2005-2008 (Continued)

\begin{tabular}{|c|c|c|c|c|c|c|}
\hline Slovakia & 2006 & $\begin{array}{c}50.1 \\
(48.5-51.8) \\
\end{array}$ & $\begin{array}{c}79.4 \\
(78.0-80.6) \\
\end{array}$ & $\begin{array}{c}85.2 \\
(84.0-86.3) \\
\end{array}$ & $\begin{array}{c}48.2 \\
(46.4-50.0) \\
\end{array}$ & $\begin{array}{c}59.6 \\
(55.9-63.2) \\
\end{array}$ \\
\hline Slovenia & 2007 & $\begin{array}{c}28.2 \\
(24.1-32.6) \\
\end{array}$ & $\begin{array}{c}76.8 \\
(72.5-80.5) \\
\end{array}$ & $\begin{array}{c}90.1 \\
(86.9-92.6) \\
\end{array}$ & $\begin{array}{c}97.6 \\
(95.5-98.7) \\
\end{array}$ & $\begin{array}{c}53.3 \\
(39.0-67.1) \\
\end{array}$ \\
\hline \multicolumn{7}{|c|}{ REGION OF THE AMERICAS (AMR) } \\
\hline Argentina & 2005 & $\begin{array}{c}65.4 \\
(63.5-67.2) \\
\end{array}$ & $\begin{array}{c}91.3 \\
(90.1-92.3) \\
\end{array}$ & $\begin{array}{c}69.0 \\
(66.8-71.1) \\
\end{array}$ & $\begin{array}{c}78.8 \\
(76.3-81.1) \\
\end{array}$ & $\begin{array}{c}62.2 \\
(58.5-65.7) \\
\end{array}$ \\
\hline Bolivia & 2006 & $\begin{array}{c}46.8 \\
(41.6-52.1)\end{array}$ & $\begin{array}{c}74.2 \\
(69.2-78.6)\end{array}$ & $\begin{array}{c}34.4 \\
(21.1-50.7)\end{array}$ & $\begin{array}{c}61.4 \\
(44.8-75.8)\end{array}$ & $\begin{array}{c}65.7 \\
(60.8-70.3)\end{array}$ \\
\hline $\begin{array}{l}\text { Brazil (Rio } \\
\text { de Janeiro) }\end{array}$ & 2006 & $\begin{array}{c}24.3 \\
(22.8-25.7)\end{array}$ & $\begin{array}{c}75.5 \\
(74.0-76.9)\end{array}$ & $\begin{array}{c}8.8 \\
(7.5-10.2)\end{array}$ & $\begin{array}{c}5.6 \\
(3.0-10.5)\end{array}$ & $\begin{array}{c}49.1 \\
(43.3-54.8)\end{array}$ \\
\hline Chile & 2008 & $\begin{array}{c}42.2 \\
(40.8-43.7)\end{array}$ & $\begin{array}{c}83.2 \\
(82.1-84.3)\end{array}$ & $\begin{array}{c}67.5 \\
(66.0-69.1)\end{array}$ & $\begin{array}{c}57.6 \\
(55.3-59.8)\end{array}$ & $\begin{array}{c}57.7 \\
(54.7-60.7)\end{array}$ \\
\hline Costa Rica & 2006 & 45.9 & 87.7 & 69.9 & 75.3 & 65.6 \\
\hline $\begin{array}{l}\text { Cuba } \\
\text { (Havana) }\end{array}$ & 2008 & $\begin{array}{c}71.3 \\
(69.4-73.2)\end{array}$ & $\begin{array}{c}91.8 \\
(90.7-92.9)\end{array}$ & $\begin{array}{c}60.8 \\
(58.7-62.8)\end{array}$ & $\begin{array}{c}31.4 \\
(29.0-33.9)\end{array}$ & $\begin{array}{c}58.2 \\
(54.1-62.1)\end{array}$ \\
\hline Guatemala & 2008 & $\begin{array}{c}34.6 \\
(31.5-37.8) \\
\end{array}$ & $\begin{array}{c}75.0 \\
(72.0-77.7) \\
\end{array}$ & $\begin{array}{c}72.7 \\
(69.7-75.6) \\
\end{array}$ & $\begin{array}{c}19.4 \\
(16.4-22.8) \\
\end{array}$ & $\begin{array}{c}67.7 \\
(58.4-75.9) \\
\end{array}$ \\
\hline Jamaica & 2008 & $\begin{array}{c}29.0 \\
(24.1-34.4) \\
\end{array}$ & $\begin{array}{c}58.9 \\
(53.2-64.4) \\
\end{array}$ & $\begin{array}{c}82.1 \\
(77.2-86.1) \\
\end{array}$ & $\begin{array}{c}50.6 \\
(44.1-57.0) \\
\end{array}$ & $*$ \\
\hline Mexico & 2006 & $\begin{array}{c}46.8 \\
(40.4-53.2) \\
\end{array}$ & $\begin{array}{c}83.7 \\
(79.3-87.3) \\
\end{array}$ & $\begin{array}{c}52.3 \\
(42.3-62.1) \\
\end{array}$ & $\begin{array}{c}62.1 \\
(51.3-71.8) \\
\end{array}$ & $\begin{array}{c}58.4 \\
(52.0-64.5) \\
\end{array}$ \\
\hline Panama & 2008 & $\begin{array}{c}29.7 \\
(27.4-32.2) \\
\end{array}$ & $\begin{array}{c}51.1 \\
(48.5-53.7) \\
\end{array}$ & $\begin{array}{c}75.3 \\
(72.9-77.5) \\
\end{array}$ & $\begin{array}{c}66.1 \\
(63.2-68.9) \\
\end{array}$ & $\begin{array}{c}69.4 \\
(60.2-77.3) \\
\end{array}$ \\
\hline Paraguay & 2008 & $\begin{array}{c}46.4 \\
(44.7-48.2)\end{array}$ & $\begin{array}{c}82.5 \\
(81.1-83.7)\end{array}$ & $\begin{array}{c}26.1 \\
(24.6-27.7)\end{array}$ & $\begin{array}{c}44.7 \\
(41.3-48.1)\end{array}$ & $\begin{array}{c}86.0 \\
(82.3-89.1)\end{array}$ \\
\hline Peru & 2006 & $\begin{array}{c}37.1 \\
(31.0-43.6)\end{array}$ & $\begin{array}{c}65.4 \\
(60.4-70.2)\end{array}$ & $\begin{array}{c}31.2 \\
(20.7-44.1)\end{array}$ & $\begin{array}{c}59.0 \\
(48.9-68.3)\end{array}$ & $\begin{array}{c}59.7 \\
(52.7-66.3)\end{array}$ \\
\hline Uruguay & 2008 & $\begin{array}{c}39.7 \\
(38.6-40.7) \\
\end{array}$ & $\begin{array}{c}49.1 \\
(48.0-50.2) \\
\end{array}$ & $\begin{array}{c}90.7 \\
(90.0-91.3) \\
\end{array}$ & $\begin{array}{c}91.3 \\
(90.6-91.9) \\
\end{array}$ & $\begin{array}{c}53.7 \\
(51.4-56.0)\end{array}$ \\
\hline \multicolumn{7}{|c|}{ SOUTH-EAST ASIA REGION (SEAR) } \\
\hline Bangladesh & 2006 & $\begin{array}{c}44.7 \\
(24.9-66.3) \\
\end{array}$ & $\begin{array}{c}77.1 \\
(45.9-93.1) \\
\end{array}$ & $\begin{array}{c}67.2 \\
(40.7-86.0) \\
\end{array}$ & $\begin{array}{c}79.1 \\
(51.8-93.0) \\
\end{array}$ & $\begin{array}{c}26.6 \\
(12.4-48.1) \\
\end{array}$ \\
\hline India & 2005 & $\begin{array}{c}42.8 \\
(37.9-47.9)\end{array}$ & $\begin{array}{c}73.7 \\
(68.9-78.0)\end{array}$ & $\begin{array}{c}48.0 \\
(44.5-51.5)\end{array}$ & $\begin{array}{c}62.8 \\
(54.7-70.3)\end{array}$ & $\begin{array}{c}71.8 \\
(52.7-85.4)\end{array}$ \\
\hline Indonesia & 2006 & $\begin{array}{c}44.6 \\
(37.9-51.4) \\
\end{array}$ & $\begin{array}{c}80.3 \\
(72.3-86.3) \\
\end{array}$ & $\begin{array}{c}41.1 \\
(24.7-59.8) \\
\end{array}$ & $\begin{array}{c}41.0 \\
(29.5-53.6) \\
\end{array}$ & $\begin{array}{c}76.6 \\
(53.1-90.4)\end{array}$ \\
\hline Myanmar & 2006 & $\begin{array}{c}54.4 \\
(53.4-55.4) \\
\end{array}$ & $\begin{array}{c}78.5 \\
(77.6-79.3) \\
\end{array}$ & $\begin{array}{c}68.1 \\
(67.2-69.1) \\
\end{array}$ & $\begin{array}{c}52.8 \\
(51.5-54.1) \\
\end{array}$ & $\begin{array}{c}74.8 \\
(71.3-78.0) \\
\end{array}$ \\
\hline Nepal & 2005 & $\begin{array}{c}45.9 \\
(26.1-67.0)\end{array}$ & $\begin{array}{c}69.3 \\
(48.3-84.5)\end{array}$ & $\begin{array}{c}53.1 \\
(47.2-59.0)\end{array}$ & $\begin{array}{c}56.8 \\
(33.3-77.5)\end{array}$ & $\begin{array}{c}65.3 \\
(32.0-88.3)\end{array}$ \\
\hline Sri Lanka & 2006 & $\begin{array}{c}21.1 \\
(19.6-22.7)\end{array}$ & $\begin{array}{c}54.5 \\
(52.5-56.4)\end{array}$ & $\begin{array}{c}39.4 \\
(37.4-41.4)\end{array}$ & $\begin{array}{c}74.2 \\
(70.9-77.3)\end{array}$ & $\begin{array}{c}66.7 \\
(55.2-76.5)\end{array}$ \\
\hline Thailand & 2006 & $\begin{array}{c}27.3 \\
(25.0-29.7) \\
\end{array}$ & $\begin{array}{c}56.1 \\
(53.5-58.6) \\
\end{array}$ & $\begin{array}{c}48.4 \\
(45.8-51.0) \\
\end{array}$ & $\begin{array}{c}80.0 \\
(76.5-83.1)\end{array}$ & $\begin{array}{c}69.5 \\
(46.1-85.8)\end{array}$ \\
\hline \multicolumn{7}{|c|}{ WESTERN PACIFIC REGION (WPR) } \\
\hline Cambodia & 2005 & $\begin{array}{c}57.7 \\
(52.1-63.0) \\
\end{array}$ & $\begin{array}{c}64.0 \\
(58.5-69.1) \\
\end{array}$ & $\begin{array}{c}63.1 \\
(57.5-68.3) \\
\end{array}$ & $\begin{array}{c}73.1 \\
(65.3-79.7) \\
\end{array}$ & * \\
\hline South Korea & 2006 & $\begin{array}{c}16.8 \\
(14.4-19.5) \\
\end{array}$ & $\begin{array}{c}75.2 \\
(69.5-80.1) \\
\end{array}$ & $\begin{array}{c}78.6 \\
(72.5-83.7) \\
\end{array}$ & $\begin{array}{c}83.4 \\
(79.0-87.0) \\
\end{array}$ & $\begin{array}{c}65.5 \\
(53.6-75.7) \\
\end{array}$ \\
\hline Viet Nam & 2007 & $\begin{array}{c}61.4 \\
(60.6-62.3)\end{array}$ & $\begin{array}{c}71.2 \\
(70.4-72.0)\end{array}$ & $\begin{array}{c}71.8 \\
(71.0-72.6)\end{array}$ & $\begin{array}{c}88.2 \\
(87.3-89.1)\end{array}$ & $\begin{array}{c}71.1 \\
(68.0-74.1)\end{array}$ \\
\hline
\end{tabular}

${ }^{*}$ Cell size less than 10 . 
Table 5 Attitudes Toward and Training in Patient Smoking Cessation Counseling, by Region and Country, Medical Global Health Professions Student Survey, 2005-2008

\begin{tabular}{|c|c|c|c|c|}
\hline & & $\begin{array}{l}\text { Thought health professionals have a } \\
\text { role in giving advice or information } \\
\text { about smoking cessation to patients }\end{array}$ & $\begin{array}{l}\text { Thought health professionals } \\
\text { should get specific training } \\
\text { on cessation techniques }\end{array}$ & $\begin{array}{l}\text { Had ever received any formal training in } \\
\text { smoking cessation approaches to use with } \\
\text { patients in their medical school training }\end{array}$ \\
\hline $\begin{array}{l}\text { Country } \\
\text { (Site) }\end{array}$ & Year & $\begin{array}{l}\text { Total \% } \\
(95 \% \mathrm{Cl})\end{array}$ & $\begin{array}{l}\text { Total \% } \\
(95 \% \mathrm{Cl})\end{array}$ & $\begin{array}{l}\text { Total \% } \\
(95 \% \mathrm{Cl})\end{array}$ \\
\hline \multicolumn{5}{|c|}{ AFRICAN REGION (AFR) } \\
\hline Algeria & 2007 & $\begin{array}{c}85.6 \\
(84.6-86.7)\end{array}$ & $\begin{array}{c}89.7 \\
(88.7-90.5)\end{array}$ & $\begin{array}{c}36.1 \\
(34.7-37.5)\end{array}$ \\
\hline Kenya & 2008 & $\begin{array}{c}98.2 \\
(96.8-99.0)\end{array}$ & $\begin{array}{c}96.9 \\
(95.2-98.0)\end{array}$ & $\begin{array}{c}26.0 \\
(22.7-29.6)\end{array}$ \\
\hline Niger & 2008 & 100.0 & $\begin{array}{c}97.2 \\
(96.6-97.8)\end{array}$ & $\begin{array}{c}46.4 \\
(44.7-48.1)\end{array}$ \\
\hline Uganda & 2005 & $\begin{array}{c}98.8 \\
(97.7-99.3)\end{array}$ & $\begin{array}{c}97.3 \\
(95.9-98.2)\end{array}$ & $\begin{array}{c}15.9 \\
(13.5-18.6)\end{array}$ \\
\hline \multicolumn{5}{|c|}{ EASTERN MEDITERRANEAN REGION (EMR) } \\
\hline Egypt & 2005 & $\begin{array}{c}91.1 \\
(89.6-92.4)\end{array}$ & $\begin{array}{c}92.5 \\
(90.4-94.2)\end{array}$ & $\begin{array}{c}20.9 \\
(18.4-23.6)\end{array}$ \\
\hline $\begin{array}{l}\text { Gaza Strip/ } \\
\text { West Bank }\end{array}$ & 2007 & $\begin{array}{c}88.4 \\
(85.7-90.6)\end{array}$ & $\begin{array}{c}95.1 \\
(93.3-96.5)\end{array}$ & $\begin{array}{c}25.6 \\
(22.5-28.9)\end{array}$ \\
\hline $\operatorname{Iran}$ & 2007 & $\begin{array}{c}91.7 \\
(90.1-93.1) \\
\end{array}$ & $\begin{array}{c}94.8 \\
(93.5-95.8)\end{array}$ & $\begin{array}{c}10.0 \\
(8.4-11.8)\end{array}$ \\
\hline Iraq & 2005 & $\begin{array}{c}63.8 \\
(61.0-66.5)\end{array}$ & $\begin{array}{c}77.9 \\
(75.4-80.2)\end{array}$ & $\begin{array}{c}31.3 \\
(28.7-34.0)\end{array}$ \\
\hline Lebanon & 2006 & $\begin{array}{c}88.9 \\
(86.7-90.8)\end{array}$ & $\begin{array}{c}94.0 \\
(92.1-95.4)\end{array}$ & $\begin{array}{c}29.7 \\
(26.7-33.0)\end{array}$ \\
\hline $\begin{array}{l}\text { Libyan Arab } \\
\text { Jamahiriya }\end{array}$ & 2006 & $\begin{array}{c}77.4 \\
(76.0-78.8)\end{array}$ & $\begin{array}{c}87.1 \\
(85.9-88.1)\end{array}$ & $\begin{array}{c}24.9 \\
(23.4-26.3)\end{array}$ \\
\hline $\begin{array}{l}\text { Saudi } \\
\text { Arabia }\end{array}$ & 2006 & $\begin{array}{c}97.8 \\
(96.3-98.7)\end{array}$ & $\begin{array}{c}89.1 \\
(86.4-91.4)\end{array}$ & $\begin{array}{c}6.4 \\
(4.6-8.7)\end{array}$ \\
\hline Sudan & 2007 & $\begin{array}{c}97.9 \\
(96.8-98.6) \\
\end{array}$ & $\begin{array}{c}97.5 \\
(96.4-98.3) \\
\end{array}$ & $\begin{array}{c}31.6 \\
(28.9-34.5) \\
\end{array}$ \\
\hline $\begin{array}{l}\text { Syrian Arab } \\
\text { Republic } \\
\end{array}$ & 2006 & $\begin{array}{c}95.7 \\
(95.3-96.0) \\
\end{array}$ & $\begin{array}{c}96.8 \\
(96.5-97.1) \\
\end{array}$ & $\begin{array}{c}29.4 \\
(28.5-30.2) \\
\end{array}$ \\
\hline Tunisia & 2007 & $\begin{array}{c}96.5 \\
(95.5-97.3) \\
\end{array}$ & $\begin{array}{c}94.0 \\
(92.8-95.1) \\
\end{array}$ & $\begin{array}{c}23.0 \\
(20.9-25.1) \\
\end{array}$ \\
\hline \multicolumn{5}{|c|}{ EUROPEAN REGION (EUR) } \\
\hline Albania & 2005 & $\begin{array}{c}95.0 \\
(93.8-95.9) \\
\end{array}$ & $\begin{array}{c}97.1 \\
(96.2-97.8) \\
\end{array}$ & $\begin{array}{c}10.3 \\
(9.0-11.9) \\
\end{array}$ \\
\hline Armenia & 2006 & $\begin{array}{c}80.8 \\
(75.8-85.0) \\
\end{array}$ & $\begin{array}{c}84.4 \\
(79.7-88.2) \\
\end{array}$ & $\begin{array}{c}34.1 \\
(28.9-39.8) \\
\end{array}$ \\
\hline $\begin{array}{l}\text { Bosnia \& } \\
\text { Herzegovina }\end{array}$ & 2006 & $\begin{array}{c}86.0 \\
(85.1-86.8)\end{array}$ & $\begin{array}{c}86.4 \\
(85.5-87.1)\end{array}$ & $\begin{array}{c}11.2 \\
(10.5-12.0)\end{array}$ \\
\hline Croatia & 2005 & $\begin{array}{c}97.7 \\
(96.8-98.4)\end{array}$ & $\begin{array}{c}71.7 \\
(69.3-73.9)\end{array}$ & $\begin{array}{c}14.5 \\
(12.8-16.4)\end{array}$ \\
\hline $\begin{array}{l}\text { Czech } \\
\text { Republic }\end{array}$ & 2006 & $\begin{array}{c}82.0 \\
(79.8-84.0) \\
\end{array}$ & $\begin{array}{c}60.8 \\
(58.1-63.5)\end{array}$ & $\begin{array}{c}1.4 \\
(0.9-2.2)\end{array}$ \\
\hline Kyrgyzstan & 2008 & $\begin{array}{c}85.7 \\
(83.6-87.6)\end{array}$ & $\begin{array}{c}92.0 \\
(90.3-93.4)\end{array}$ & $\begin{array}{c}22.3 \\
(20.1-24.8)\end{array}$ \\
\hline Lithuania & 2006 & $\begin{array}{c}87.6 \\
(84.3-90.2)\end{array}$ & $\begin{array}{c}96.1 \\
(94.1-97.4)\end{array}$ & $\begin{array}{c}25.2 \\
(21.6-29.2)\end{array}$ \\
\hline $\begin{array}{l}\text { Russian } \\
\text { Federation }\end{array}$ & 2006 & NA & $\begin{array}{c}77.1 \\
(76.2-78.1)\end{array}$ & $\begin{array}{c}20.6 \\
(19.7-21.6)\end{array}$ \\
\hline Serbia & 2006 & $\begin{array}{c}89.9 \\
(88.9-90.8)\end{array}$ & $\begin{array}{c}81.5 \\
(80.2-82.7)\end{array}$ & $\begin{array}{c}21.3 \\
(20.0-22.6)\end{array}$ \\
\hline
\end{tabular}


Table 5 Attitudes Toward and Training in Patient Smoking Cessation Counseling, by Region and Country, Medical Global Health Professions Student Survey, 2005-2008 (Continued)

\begin{tabular}{|c|c|c|c|c|}
\hline Slovakia & 2006 & $\begin{array}{c}59.7 \\
(58.0-61.3) \\
\end{array}$ & $\begin{array}{c}71.4 \\
(69.9-72.8) \\
\end{array}$ & $\begin{array}{c}3.0 \\
(2.5-3.6) \\
\end{array}$ \\
\hline Slovenia & 2007 & 100.0 & $\begin{array}{c}77.5 \\
(73.3-81.2) \\
\end{array}$ & $\begin{array}{c}5.6 \\
(3.8-8.2) \\
\end{array}$ \\
\hline \multicolumn{5}{|c|}{ REGION OF THE AMERICAS (AMR) } \\
\hline Argentina & 2005 & $\begin{array}{c}98.8 \\
(98.3-99.1) \\
\end{array}$ & $\begin{array}{c}91.3 \\
(90.1-92.3) \\
\end{array}$ & $\begin{array}{c}5.2 \\
(4.4-6.1) \\
\end{array}$ \\
\hline Bolivia & 2006 & $\begin{array}{c}87.6 \\
(84.4-90.2)\end{array}$ & $\begin{array}{c}95.5 \\
(93.4-97.0)\end{array}$ & $\begin{array}{c}20.4 \\
(14.3-28.3)\end{array}$ \\
\hline $\begin{array}{l}\text { Brazil (Rio } \\
\text { de Janeiro) }\end{array}$ & 2006 & $\begin{array}{c}81.9 \\
(80.5-83.1)\end{array}$ & $\begin{array}{c}94.3 \\
(93.5-95.1)\end{array}$ & $\begin{array}{c}21.2 \\
(19.9-22.7)\end{array}$ \\
\hline Chile & 2008 & $\begin{array}{c}97.5 \\
(97.0-97.9)\end{array}$ & $\begin{array}{c}93.4 \\
(92.7-94.0)\end{array}$ & $\begin{array}{c}23.4 \\
(22.1-24.6)\end{array}$ \\
\hline Costa Rica & 2006 & 97.1 & 94.9 & 11.2 \\
\hline $\begin{array}{l}\text { Cuba } \\
\text { (Havana) }\end{array}$ & 2008 & 100.0 & $\begin{array}{c}98.7 \\
(98.2-99.1)\end{array}$ & $\begin{array}{c}28.9 \\
(27.1-30.8)\end{array}$ \\
\hline Guatemala & 2008 & 100.0 & $\begin{array}{c}96.3 \\
(94.9-97.4) \\
\end{array}$ & $\begin{array}{c}23.6 \\
(20.8-26.5) \\
\end{array}$ \\
\hline Jamaica & 2008 & $\begin{array}{c}99.1 \\
(97.0-99.7) \\
\end{array}$ & $\begin{array}{c}94.3 \\
(91.0-96.5) \\
\end{array}$ & $\begin{array}{c}8.5 \\
(5.8-12.3) \\
\end{array}$ \\
\hline Mexico & 2006 & $\begin{array}{c}85.8 \\
(83.0-88.3) \\
\end{array}$ & $\begin{array}{c}94.7 \\
(93.0-96.0) \\
\end{array}$ & $\begin{array}{c}20.4 \\
(14.5-27.8) \\
\end{array}$ \\
\hline Panama & 2008 & 100.0 & $\begin{array}{c}94.6 \\
(93.3-95.7) \\
\end{array}$ & $\begin{array}{c}26.1 \\
(23.8-28.4) \\
\end{array}$ \\
\hline Paraguay & 2008 & $\begin{array}{c}96.0 \\
(95.3-96.6)\end{array}$ & $\begin{array}{c}98.8 \\
(98.4-99.1)\end{array}$ & $\begin{array}{c}30.8 \\
(29.2-32.4)\end{array}$ \\
\hline Peru & 2006 & $\begin{array}{c}95.9 \\
(93.1-97.5)\end{array}$ & $\begin{array}{c}97.5 \\
(94.2-98.9)\end{array}$ & $\begin{array}{c}30.0 \\
(21.9-39.4)\end{array}$ \\
\hline Uruguay & 2008 & $\begin{array}{c}93.0 \\
(92.4-93.6) \\
\end{array}$ & $\begin{array}{c}89.5 \\
(88.8-90.2) \\
\end{array}$ & $\begin{array}{c}10.9 \\
(10.2-11.6) \\
\end{array}$ \\
\hline \multicolumn{5}{|c|}{ SOUTH-EAST ASIA REGION (SEAR) } \\
\hline Bangladesh & 2006 & $\begin{array}{c}71.1 \\
(45.2-88.0) \\
\end{array}$ & $\begin{array}{c}87.8 \\
(81.2-92.3) \\
\end{array}$ & $\begin{array}{c}24.7 \\
(12.2-43.6) \\
\end{array}$ \\
\hline India & 2005 & $\begin{array}{c}96.9 \\
(95.1-98.0)\end{array}$ & $\begin{array}{c}91.0 \\
(89.3-92.5)\end{array}$ & $\begin{array}{c}22.3 \\
(18.3-26.9)\end{array}$ \\
\hline Indonesia & 2006 & $\begin{array}{c}98.1 \\
(97.1-98.7) \\
\end{array}$ & $\begin{array}{c}95.8 \\
(92.9-97.5) \\
\end{array}$ & $\begin{array}{c}22.2 \\
(5.3-59.2)\end{array}$ \\
\hline Myanmar & 2006 & $\begin{array}{c}94.8 \\
(94.3-95.2) \\
\end{array}$ & $\begin{array}{c}96.4 \\
(96.0-96.8) \\
\end{array}$ & $\begin{array}{c}43.7 \\
(42.7-44.7) \\
\end{array}$ \\
\hline Nepal & 2005 & $\begin{array}{c}97.0 \\
(93.8-98.5)\end{array}$ & $\begin{array}{c}93.0 \\
(85.1-96.8)\end{array}$ & $\begin{array}{c}21.9 \\
(12.8-34.9)\end{array}$ \\
\hline Sri Lanka & 2006 & $\begin{array}{c}93.9 \\
(92.9-94.8)\end{array}$ & $\begin{array}{c}90.6 \\
(89.4-91.7)\end{array}$ & $\begin{array}{c}16.2 \\
(14.8-17.7)\end{array}$ \\
\hline Thailand & 2006 & $\begin{array}{c}95.7 \\
(94.5-96.6)\end{array}$ & $\begin{array}{c}93.1 \\
(91.7-94.3) \\
\end{array}$ & $\begin{array}{c}24.0 \\
(21.8-26.3)\end{array}$ \\
\hline \multicolumn{5}{|c|}{ WESTERN PACIFIC REGION (WPR) } \\
\hline Cambodia & 2005 & $\begin{array}{c}99.1 \\
(97.1-99.7) \\
\end{array}$ & $\begin{array}{c}99.1 \\
(97.1-99.7) \\
\end{array}$ & $\begin{array}{c}14.4 \\
(10.9-18.8) \\
\end{array}$ \\
\hline South Korea & 2006 & $\begin{array}{c}93.3 \\
(89.0-96.0) \\
\end{array}$ & $\begin{array}{c}76.6 \\
(72.6-80.2) \\
\end{array}$ & $\begin{array}{c}18.9 \\
(14.6-24.2) \\
\end{array}$ \\
\hline Viet Nam & 2007 & NA & $\begin{array}{c}92.0 \\
(91.5-92.5)\end{array}$ & $\begin{array}{c}27.2 \\
(26.4-28.0)\end{array}$ \\
\hline
\end{tabular}

NA Data not available. 
tobacco by providing encouragement and information to students who are considering quitting and providing assistance to students who are motivated to quit.

\section{Exposure to Secondhand Smoke (SHS)}

Medical schools/colleges should be encouraged to provide smoke-free work and study areas by banning smoking in their buildings and clinics. A smoke-free work environment has been shown to improve air quality, reduce health problems associated with exposure to tobacco smoke, support and encourage cessation attempts among smokers trying to quit, and receive high levels of public support from people who spend time in the area [16]. Furthermore, the creation of smoke-free areas by educational institutions sends a clear message to educators, students, patients, and clinicians about the negative impact of tobacco [17]. Results from the Medical GHPSS show high exposure to SHS: over $50 \%$ of the medical students reported they were exposed to SHS in their home in 15 of the 48 sites and over $70 \%$ were exposed to SHS in public places in 29 of the 48 sites.

\section{Health Professional Training}

Medical students should be trained to provide effective, accurate, and accessible advice to patients on all aspects of health. Results from the Medical GHPSS show that over $80 \%$ of medical students recognize that they are role models in society; over $80 \%$ think they should receive training on counseling and treating patients to quit using tobacco, but less than $40 \%$ have received formal training.

The Medical GHPSS surveyed $3^{\text {rd }}$ year students, so it is possible that students receive training on patient cessation techniques during the latter years of their programs. To address this possibility, the GHPSS research coordinators raised this question to the school administrators and found that, in the majority of the countries, there is no formal training at any time. Of the countries with some training, the type of training included: problembased learning, included in general counseling curricula; or were included in curricula as part of community medicine or public health courses. This study did not make an effort to evaluate the adequacy of cessation training in the countries reporting this type of instruction. However, professional training for medical students should include courses detailing the harmful health effects of tobacco use and exposure to SHS, and training in counseling on tobacco cessation techniques [1].

\section{Limitations}

The Medical GHPSS is subject to at least four limitations. First, this study reflects $3^{\text {rd }}$ year students who have not had substantial interaction with patients, so these survey results should not be extrapolated to account for practicing health professionals. Second, the sites including in this study are not representative of individual WHO regions given the number of sites included per region (of the 193 WHO Member States we report data for 47 sites and one geographic region). Third, since the adult smoking rates cited from the WHO Report on the Global Tobacco Epidemic (GTCR) 2008, were not collected using a standardized and consistent methodology the comparison of current cigarette smoking prevalence rates should be interpreted with caution. Lastly, data were based on the self-report of students, who might underreport or over-report their behaviors or attitudes. The extent of this bias cannot be determined from these data; however, reliability studies in the United States have indicated good test-retest results for similar tobacco-related questions [18].

\section{Conclusions}

Educational institutions, public health organizations, and education officials should discourage tobacco use among medical students and all health professionals and work together to design and implement programs that train medical students in effective cessation-counseling techniques by utilizing evidence-based strategies. Previous studies have noted the following problems in preparing medical students for a role in tobacco control: 1) a lack of tobacco-related material in medical school curricula [19-22]; and 2) practicing physicians report difficulties delivering tobacco cessation care to patients due to lack of time, reluctance to get involved in personal issues, and failure to use evidence-based methods with patients [23]. Despite these barriers, structural and institutional interventions such as creating tobacco-free hospitals and providing insurance coverage for cessation services combined with practice-level strategies such as including tobacco cessation training in expected physician competency measures could foster a positive environment for tobacco control in the healthcare delivery system. This combined approach among health institutions and medical professionals should improve the incorporation of tobacco cessation into standard healthcare delivery [24].

The Medical GHPSS has shown global gaps in medical school training to provide effective patient tobacco cessation counseling to their future patients. Tobacco control efforts in these countries should include programmatic efforts that help train medical students to deliver these counseling services that can assist people in changing their smoking behavior. This is particularly important for the sites included in this study that have ratified the WHO FCTC (43 of the 47 sites) who are legally obligated to implement the provisions of the Articles under the treaty. This study also shows strong interest on the part of the students in receiving this 
training, despite previous studies that suggest a lack of interest among students to learn about the health consequences of tobacco use [22]. The Medical GHPSS is helpful in evaluating the behavior and attitudes regarding tobacco use among medical students, but additional research is necessary to improve the evidence base for effective tobacco-related curricula, especially materials that are appropriate for a range of cultural and economic settings. Repeating the Medical GHPSS will prove useful in evaluating the effectiveness of tobaccorelated curricula for future health professionals in these countries.

\section{Other Notes}

CW Warren, J Lee and V Lea are obligated by their institution to have the following statement printed in the report: "The findings and conclusions in this report are those of the authors and do not necessarily represent the views of the Centers for Disease Control and Prevention."

\section{Acknowledgements}

The authors had full access to all of the data in the study and take responsibility for the integrity of the data and the accuracy of the data analysis. The authors would like to thank the following who made completion of the Medical GHPSS possible.

\section{${ }^{*}$ GHPSS Country Research Coordinators}

WHO Regional Offices: African Region, Jean-Pierre Baptiste, Nivo Ramanandraibe; Eastern Mediterranean Region, Fatimah El-Awa, Heba Fouad; European Region, Agis Tsouros, Kristina Mauer-Stender, Rula Nabil Khoury; Region of the Americas, Adriana Blanco, Roberta Caixeta; South-East Asia Region, Khalilur Rahman, Dhirendra N. Sinha; Western Pacific Region, Susan Mercado, Ali Akbar.

African Region: Djamel Zoughailech (Algeria); Joyce Nato, Ezra Ouma Ogwell (Kenya); Magagi Gagaara (Niger) Frederick Musoke (Uganda). Eastern Mediterranean Region: Mohamed Mehrez Mostafa (Egypt); Samah Eriqat, Salah Shaker Isa Soubani, Moein Al Kariry (Gaza Strip and West Bank); Ahmed Ali Bahaj, Ali Asghar Farshad, Hassan Azaripour Masooleh (Iran); Sarhang Jalal (Iraq); Heba Ayoub; Georges Saade, Nagib Ghosn (Lebanon); Mohamed Ibrahem Saleh (Libya); Albedah Abdullah (Saudi Arabia); Ibrahim Abdelmageed Mohamed Ginawi, Ilham Abdalla Bashir (Sudan); Bassam Abu Al Zahab (Syria); Mohamed Nabil Ben Sahem, Alya Mahjoub Zarrouk, Mohamed Mokdad, Mongi Hamrouni (Tunisia).

European Region: Roland Shuperka (Albania); Alexander Bazarjyan (Armenia); Aida Ramic-Catak, Zivana Gavric (Bosnia \& Herzegovina); Hrvoje Vrazic (Croatia);Hana Sovinova (Czech Republic); Aisha Tokobaeva (Kyrgyzstan); Antanas Gostautas (Lithuania); Galina Sakharova (Russian Federation); Djordje Stojilkovic, Andjelka Dzeletovic (Republic of Serbia); Mojca Juricic (Slovenia); Tibor Baska (Slovakia).

Region of the Americas: Raul Pitarque (Argentina); Franklin Alcaraz del Castillo (Bolivia); Luisa Goldfarb,

Valeska Caralho Figueiredo, Adelemara Mattoso Allonzi, Leticia Casado Costa, Liz Maria de Almeida (Brazil); Claudia Gonzalez Wedmaier (Chile); Julio Bejarano (Costa Rica); Lucia Lances Cotilla (Cuba); Ellen Grizzle (Jamaica); Walesska Zeceña (Guatemala); Reina Roa (Panama); Victor San Martin (Paraguay);Alfonso Zavaleta (Peru); Leo Alleyne, Raquel Magri, Gabriela Olivera (Uruguay).

South-East Asia Region: Zulfiqar Ali (Bangladesh); Dhirendra N. Sinha, Gupteshwar Singh (India); Tjandra Aditama (Indonesia); Nyo Nyo Kyaing (Myanmar); M. R. Pandey (Nepal); P. W. Gunasekera (Sri Lanka); Nithat Sirichotiratana (Thailand).

Western Pacific Region: Sin Sovann, Sung Vin Tak (Cambodia); Sun Ha Jee (South Korea) Phan Thi Hai (Vietnam).

CDC Support: Michelle Carlberg, Ann Goding, Brandon M. O'Hara.

\section{Author details}

'Office on Smoking and Health, US Centers for Disease Control and Prevention, Atlanta, GA, USA. ${ }^{2}$ Tobacco Free Initiative, South East Asia Region, World Health Organization, Delhi, India. ${ }^{3}$ Paul P. Carbone Comprehensive Cancer Center, University of Wisconsin, Madison, WI, USA.

\section{Authors' contributions}

CWW, JL, VL and NRJ conceived of the study, participated in its design and coordination, and helped to draft the manuscript. DNS participated in interpretation of the statistical analysis and drafting of the manuscript. All authors read and approved the final manuscript.

\section{Competing interests}

The authors declare that they have no competing interests.

Received: 15 July 2010 Accepted: 1 February 2011

Published: 1 February 2011

\section{References}

1. Fiore MC, Jaen CR, Baker TB, Bailey WC, Benowitz N, Curry SJ, Dorfman SF, Froelicher ES, Goldstein MG, Healton CG, Henderson PN, Heyman RB, Koh HK, Kottke TE, Lando HA, Mecklenburg RE, Mermelstein RJ, Mullen PD, Orleans CT, Robinson L, Stitzer ML, Tommasello AC, Villejo L, Wewers ME: Treating tobacco use and dependence: 2008 update. Rockville, MD U.S. Department of Health and Human Services, U.S Public Health Service; 2008.

2. World Health Organization: MPOWER: A Policy Package to Reverse the Tobacco Epidemic Geneva: World Health Organization; 2008.

3. Fang CY, Ma GX, Miller SM, Tan Y, Su X, Shive S: A brief smoking cessation intervention for Chinese and Korean American smokers. Prev Med 2006, 43(4):321-324.

4. Ferreira-Borges C: Effectiveness of a brief counseling and behavioral intervention for smoking cessation in pregnant women. Prev Med 2005, 41(1):295-302.

5. Lancaster T, Stead L: Physician advice for smoking cessation. Cochrane Database Syst Rev 2004, , 4: CD000165.

6. Molyneux A, Lewis S, Leivers U, Anderton A, Antoniak M, Brackenridge A, Nilsson F, McNeill A, West R, Moxham J, Britton J: Clinical trial comparing nicotine replacement therapy (NRT) plus brief counseling, brief counseling alone, and minimal intervention on smoking cessation in hospital inpatients. Thorax 2003, 58(6):484-488.

7. World Health Organization: WHO Framework Convention on Tobacco Control. Geneva: World Health Organization; 2003.

8. Smith DR, Leggat PA: An international review of tobacco smoking among medical students. J Postgrad Med 2007, 53(1):55-62.

9. Warren CW, Jones NR, Chauvin J, Peruga A: Tobacco use and cessation counseling: Cross-country data from the Global Health Professions Student Survey (GHPSS), 2005-7. Tob Control 2008, 17:238-247.

10. Shah BV, Barnwell BG, Bieler GS: Software for the Statistical Analysis of Correlated Data (SUDAAN): User's Manual Research Triangle Park, NC: Research Triangle Institute; 1997, Release 7.5. 1997 (software documentation).

11. Donner A, Klar N: Design and Analysis of Cluster Randomization Trials in Health Research New York, NY: Oxford University Press Inc; 2000.

12. Hinkle DE, Wiersma W, Jurs SG: Applied Statistics for the Behavioral Sciences. 5 edition. Boston, MA: Houghton Mifflin Company; 2003.

13. Vakeflliu Y, Argjiri D, Poposhi I, Agron S, Melani AS: Tobacco smoking habits, beliefs, and attitudes among medical students in Tirana, Albania. Prev Med 2002, 34:370-3.

14. Frank E: Physician health and patient care. JAMA 2004, 291:634.

15. Duncan C, Cumming SR, Hudes ES, Zahnd E, Coates TJ: Quitting smoking: reasons for quitting and predictor of cessation among medical patients. J Gen Intern Med 1992, 7:398-404.

16. Nakashima M, Miura K, Morikawa Y, Nishijo M, Nakanishi Y, Sakurai M, Nakagawa H: Effect of smoke-free medical school on smoking behavior of medical students. Japanese J Pub Health 2008, 55(9):647-54.

17. US Department of Health and Human Services, Centers for Disease Control and Prevention, Division of Nutrition, Physical Activity, Obesity: Implementing a Tobacco-Free Campus Initiative in Your Workplace. 2007 [http://www.cdc.gov/tobacco/global/].

18. Brener ND, Kann L, McMannus T, Kinchen SA, Sundberg EC, Ross JG: Reliability of the 1999 Youth Risk Behavior Survey questionnaire. J Adolesc Health 2002, 31:336-42. 
19. Davis RM: When doctors smoke. Tob Control 1993, 2:187-188.

20. Fowler G: Educating doctors in smoking cessation. Tob Control 1993, 2:5-6.

21. Nett LM: The physician's role in smoking cessation: A present and future agenda. Chest 1990, 97:28S-32S.

22. Richmond R: Teaching medical students about tobacco. Thorax 1999, 54(1):70-78.

23. Richmond RL, Zwar NA: Role of the general practitioner in smoking cessation. Drug and Alcohol Review 2006, 25:21-26.

24. Fiore MC, Keller PA, Curry SJ: Health system changes to facilitate the delivery of tobacco-dependence treatment. Am J Prev Med 2007, 33(6 Sippl):S349-S356.

\section{Pre-publication history}

The pre-publication history for this paper can be accessed here: http://www.biomedcentral.com/1471-2458/11/72/prepub

doi:10.1186/1471-2458-11-72

Cite this article as: Warren et al:: Tobacco use, exposure to secondhand smoke, and cessation counseling among medical students: cross-country data from the Global Health Professions Student Survey (GHPSS), 20052008. BMC Public Health 2011 11:72.

\section{Submit your next manuscript to BioMed Central} and take full advantage of:

- Convenient online submission

- Thorough peer review

- No space constraints or color figure charges

- Immediate publication on acceptance

- Inclusion in PubMed, CAS, Scopus and Google Scholar

- Research which is freely available for redistribution

Submit your manuscript at www.biomedcentral.com/submit 